\title{
Cross Validation of the Structure of a Transiently Formed and Low Populated FF Domain Folding Intermediate Determined by Relaxation Dispersion NMR and CS- Rosetta
}

by

Julia Audrey Barette

A thesis submitted in conformity with the requirements

for the degree of Master of Science

Graduate Department of Biochemistry

University of Toronto

(C) Copyright by Julia Audrey Barette, 2011 


\title{
Cross Validation of the Structure of a Transiently Formed and Low Populated FF Domain Folding Intermediate Determined by Relaxation Dispersion NMR and CS-Rosetta
}

\author{
Julia Audrey Barette \\ Masters of Science \\ Graduate Department of Biochemistry \\ University of Toronto
}

2011

\begin{abstract}
The atomic resolution structure of a low populated and transiently formed on-pathway folding intermediate of the FF domain from human HYPA/FBP11 has recently been reported[1]. The structure was determined on the basis of backbone chemical shift and bond vector orientation restraints measured on the 'invisible' intermediate state using relaxation dispersion nuclear magnetic resonance (NMR) spectroscopy that were subsequently input into the data-base structure determination program CS-Rosetta. This thesis focuses on the cross-validation of the structure so produced. We present here the solution NMR structure of a mimic of the folding intermediate that is highly populated in solution, obtained from the wild-type domain by protein mutagenesis. The ensemble of structures generated of the mimic are within $2 \AA$ of the relaxation dispersion/CS-Rosetta structures of the intermediate, with the non-native interactions in the intermediate also observed in the mimic. The results presented in this thesis strongly confirm the structure of the FF domain folding intermediate, in particular, and validate the use of relaxation dispersion derived restraints in structural studies of invisible excited states, in general.
\end{abstract}




\section{Table of Contents}

Chapter 1: Introduction.................................................................. 1

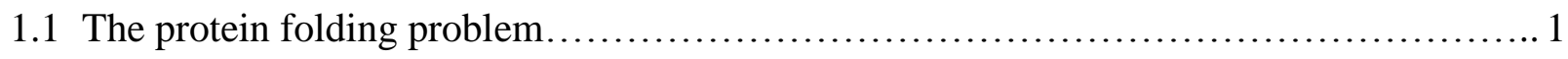

1.1.1 Models of protein folding .................................................... 1

1.1.2 Transiently formed on-pathway intermediates in protein folding .................... 3

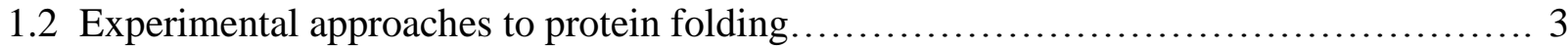

1.2.1 Experimental methods for studying folding intermediates.......................... 4

1.2.2 CPMG Relaxation dispersion NMR spectroscopy ................................ 5

1.2.3 Protein structure calculation by relaxation dispersion NMR ........................ 5

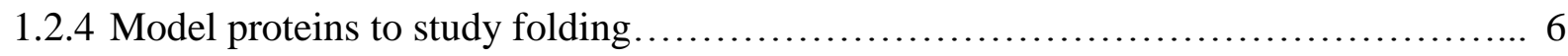

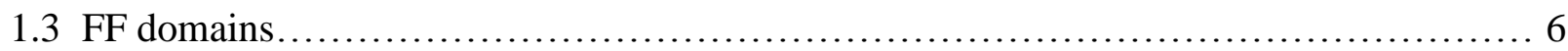

1.3.1 FF domains bind to the CTD of RNA polymerase II.............................. 6

1.3.2 The FF domain from human HYPA/FBP11folds via an on-pathway intermediate...... 7

1.3.3 The structure of the FF intermediate by relaxation dispersion NMR .................. 8

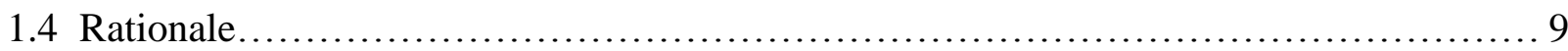

Chapter 2: Materials and methods.........................................................11

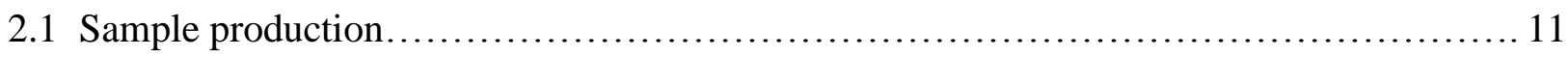

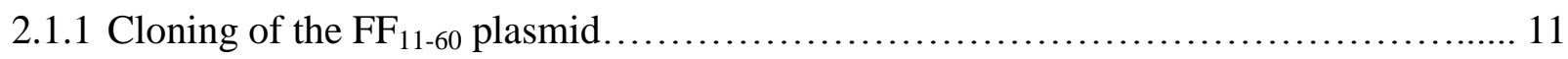

2.1.2 Protein expression.............................................................. 11

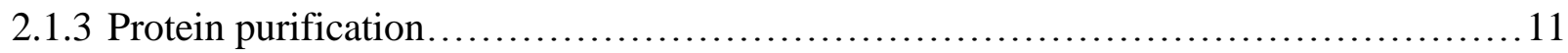

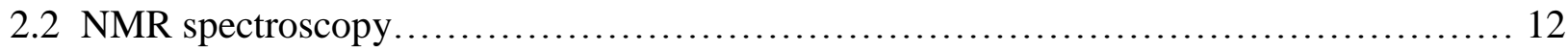

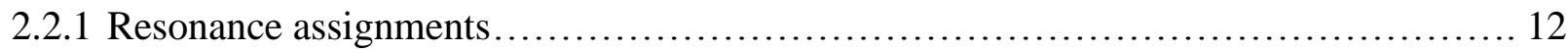

2.2.2 Stereospecific Leu and Val methyl assignments.................................... 12

2.2.3 NOESY distance restraints..................................................... 13

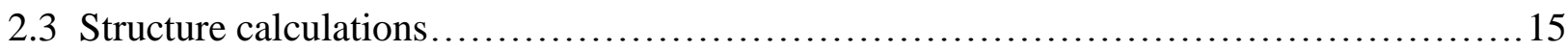


2.3.1 $\mathrm{TALOS}+\varphi / \psi$ dihedral angle prediction.................................... 15

2.3.2 RCI order parameter prediction.......................................... 16

2.3.3 XPLOR-NIH structure calculation.......................................... 16

Chapter 3: Results and discussion................................................... 18

3.1. Searching for an I state mimic as a quantitative test of structure $\ldots \ldots \ldots \ldots \ldots \ldots \ldots \ldots \ldots$

$3.2 \mathrm{FF}_{11-60}$ is a good mimic of the FF folding intermediate............................... 19

$3.3 \mathrm{I}$ and $\mathrm{I}^{\prime}$ have similar secondary structures, but $\mathrm{I}^{\prime}$ is more dynamic $\ldots \ldots \ldots \ldots \ldots \ldots \ldots \ldots . \ldots \ldots$

$3.4 \mathrm{I}^{\prime}$ forms non-native contacts predicted by the CS-Rosetta structure of I............... 22

3.5 The solution structure of I' validates the CS-Rosetta structure of I................... 24

Chapter 4: Conclusion.................................................................26

4.1 Validation of the relaxation dispersion method of solving excited state structures......... 26

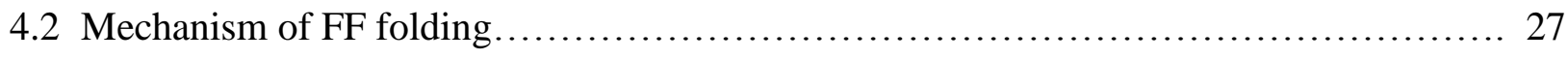

4.3 Non-native interactions in the FF folding intermediate........................... 28

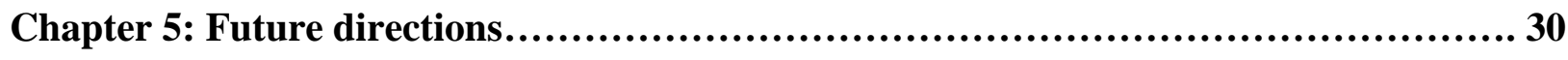




\section{List of Figures}

Figure 1: Free energy diagram view of the folding landscape $\ldots \ldots \ldots \ldots \ldots \ldots \ldots \ldots \ldots \ldots \ldots 2$

Figure 2: Structure and packing in the FF domain native state and folding intermediate....... 7

Figure 3: Stereospecific assignment of Leu $\delta$ and Val $\gamma$ methyl groups...................... 13

Figure 4: Pulse scheme for measurement of NOEs from Ala residues..................... 14

Figure 5: Effect of the A51L mutation on the methyl region of the ${ }^{1} \mathrm{H}_{-}{ }^{13} \mathrm{C}$ HSQC spectrum... 15

Figure 6: Structural statistics for the ensemble of 10 best structures with the lowest NOE and dihedral angle violations out of 100 calculated................................. 17

Figure 7: Structures and relative free energies of the unfolded, intermediate and native states of $\mathrm{FF}_{1-71}$ and of the unfolded and intermediate-like states of $\mathrm{FF}_{11-60} \ldots \ldots \ldots \ldots \ldots \ldots \ldots$

Figure 8: The $\mathrm{I}^{\prime}$ state of $\mathrm{FF}_{11-60}$ is a good mimic of the $\mathrm{FF}_{1-71}$ folding intermediate............ 20

Figure 9: Comparison of per-residue helical propensities and backbone dynamics of I' with those of the $\mathrm{FF}_{1-71}$ folding intermediate, I.......................................... 21

Figure 10: Non-native interactions predicted from the NMR relaxation dispersion/ CS-Rosetta structure of I are present in I'.

Figure 11: Solution structure of the FF domain folding intermediate mimic, $\mathrm{I}^{\prime} \ldots \ldots \ldots \ldots \ldots . . .25$

Figure 12: Per-residue helical propensity for the FF domain as predicted by AGADIR....... 28

Figure 13: Examples of proteins and their mutants that display the appropriate range of parameters for relaxation dispersion studies. 


\section{Chapter 1 \\ Introduction}

Half a century ago, the laboratory of Christian Anfinsen demonstrated that the protein ribonuclease A can fold independently of any cellular machinery[2]. This was a revolutionary discovery as it revealed that all of the information required for a protein to fold to its native state is contained in its primary amino acid sequence[3]. With this in mind, Cyrus Levinthal postulated his famous paradox that if an unfolded polypeptide chain had to sample every possible conformation allowed by its primary sequence, protein folding would take longer than the age of the universe[4]. Levinthal's solution to the paradox: protein folding is not a random process, but instead proceeds by some directed mechanism[5]. Indeed, it appears that many of the proteins whose folding has been studied to date follow directed pathways involving the transient accumulation of specific intermediate states with defined structure[1, 6-10]. In principle, structural studies of folding intermediates would provide a wealth of information about the folding process[1,10] and potentially also shed light as to how proteins misfold[11]. This thesis will focus on the structural characterisation of one such intermediate state, the on-pathway folding intermediate from the FF domain of human HYPA/FBP11.

\subsection{The protein folding problem}

Anfisen and Levinthal left us with a pair of substantial problems: how does a protein's primary sequence determine its folding pathway and final native structure, and how do proteins fold in such a short amount of time. These two questions are known as the protein folding problem. Here, we will address the second.

\subsubsection{Models of protein folding}

The earliest devised solution to Levinthal's paradox was the idea that folding proceeds along one or more defined pathways involving discrete intermediate forms[12]. Pathway specificity ensures that proteins reach their native state without having to search through large areas of conformational space. Such pathways can be simplistically illustrated as one-dimensional freeenergy diagrams as in Figure 1. Clearly, this view of folding has merit, as more and more proteins are being shown to fold via on-pathway intermediate states with defined structures (reviewed in [12-15]). However, it must be remembered that the states along the pathway retain 
considerable conformational freedom. The term "state" can be defined as an ensemble of structures with similar energy that cannot be distinguished[16]. The native state ensemble (N) is tight and displays low conformational variability. The unfolded state ensemble (U) is broad and comprises a wide range of conformations with varying amounts of residual structure. Folding intermediate states (I) fall somewhere in between. From this concept of structural heterogeneity emerged a new of view protein folding: the free energy landscape theory (reviewed in [17]). The free energy surface is a multidimensional representation of the free energy of the whole system plotted with respect to one or two physically relevant coordinates such as radius of gyration, number of native contacts or solvent-accessible surface area. The landscape displays significant ruggedness and local free-energy minima on the landscape correspond to transient kinetic traps, with the native state lying at the bottom of a set of convergent folding pathways. Despite the increased complexity of the free energy landscape, the energetics of folding can still be adequately conveyed in a one-dimensional sense as in Figure 1. The wells provide a measure of stability by showing the relative free energies of states along the pathway, the height of the energy barriers indicate their interconversion rates, and the reaction coordinate follows the folding pathway as it progresses.

(a)

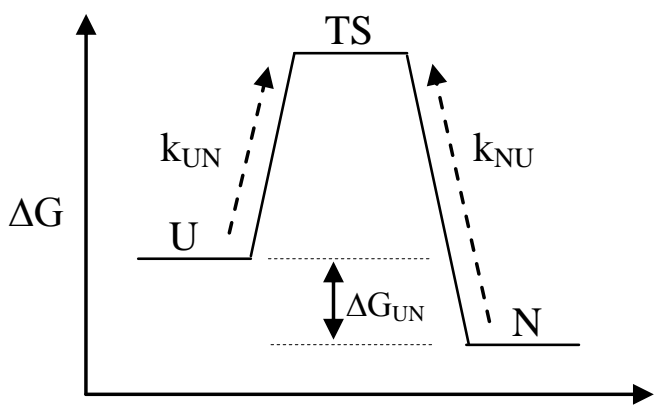

(b)

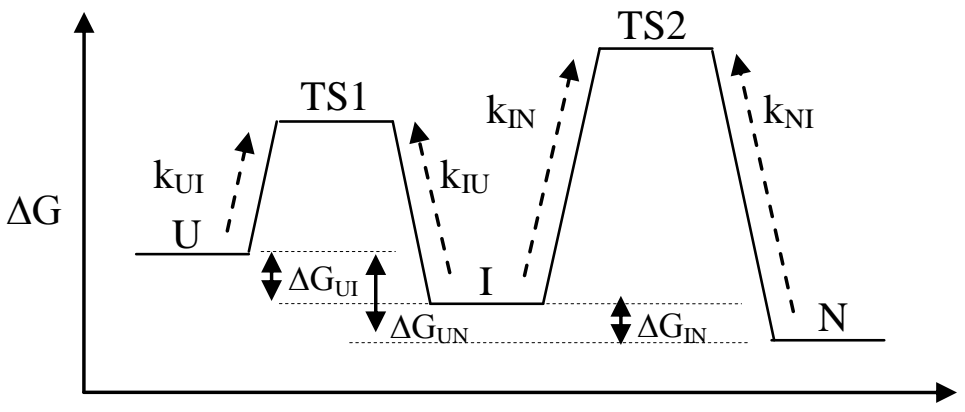

Figure 1. Free energy diagram view of the folding landscape. One-dimensional representation of a multidimensional energy surface, with free energy $(\Delta \mathrm{G})$ along the $\mathrm{y}$-axis and the reaction coordinate along the $\mathrm{x}$-axis. $\mathrm{U}$ indicates the unfolded state, I is an on-pathway intermediate state, TS, TS1 and TS2 are transition states and N is the native state. (a) Two-state folding. Only $\mathrm{U}$ and $\mathrm{N}$ are significantly populated. $\mathrm{k}_{\mathrm{UN}}$ is the rate of folding, and $\mathrm{k}_{\mathrm{NU}}$ of unfolding. $\Delta \mathrm{G}_{\mathrm{UN}}$ is the free energy difference between the unfolded and native states (typically $5-15 \mathrm{kcal} / \mathrm{mol}$ ) (b) Three-state folding. Folding proceeds via an on-pathway intermediate, I. An initial barrier TS1 precedes formation of I and a second barrier, TS2, precedes formation of the native state. $\mathrm{k}_{\mathrm{UI}}, \mathrm{k}_{\mathrm{IU}}, \mathrm{k}_{\mathrm{IN}}$ and $\mathrm{k}_{\mathrm{NI}}$ indicate the rates of interconversion between states, while $\Delta \mathrm{G}_{\mathrm{UI}}, \Delta \mathrm{G}_{\mathrm{UN}}$ and $\Delta \mathrm{G}_{\mathrm{IN}}$ indicate the free-energy differences between states.

A further level of complexity lies in the mechanism by which the conformational freedom of the polypeptide is restricted en route to the native state. The hydrophobic collapse model postulates that the hydrophobic effect drives an initial non-specific collapse, and that secondary structure 
forms later[18]. The framework model suggests that protein folding is hierarchical with secondary structure elements forming first and becoming the framework for the formation of tertiary interactions[12]. Finally, the nucleation-condensation model states that a region of the polypeptide chain serves as a folding nucleus, and that nucleation reduces the number of searches in the remainder of the polypeptide for the native structure[19]. These and several other theories are able to explain the folding behavior of some proteins, but are inconsistent with folding data for others.

\subsubsection{Transiently formed on-pathway intermediates in protein folding}

Although some small proteins are thought to fold by a 2-state mechanism in which the unfolded state transitions in a highly cooperative manner to the folded conformer[20] (Figure 1a), it is becoming increasingly clear that for many proteins, folding involves the formation of one or more transiently formed intermediates[1, 6-10, 21] (Figure 1b). Sufficiently stable intermediates can be detected kinetically using stop-flow or continuous-flow techniques and proven to be onpathway by kinetic modeling[8, 9, 22], however this is no trivial task. At equilibrium, although proteins predominantly occupy their lowest energy native state, they continue to populate all of their higher-energy forms with populations according to the Boltzmann distribution. This means that proteins under native conditions continuously unfold and refold, repeatedly revisiting their folding intermediate and unfolded states. Therefore, a sufficiently stable intermediate state may be populated to a level that is detectable at equilibrium by thermodynamic experiments, and in amenable cases be proven to be on-pathway by relaxation dispersion NMR, a technique which will be described in detail in later sections.

\subsection{Experimental approaches to protein folding}

Although we can visualize folding as following a pathway such as the ones depicted in Figure 1, it is important to remember that $\mathrm{U}, \mathrm{I}$ and $\mathrm{N}$ are all states, or conformational ensembles, not single structures. The role of the experimentalist is to characterize the relative energies, rates of conversion and structure of as many states as possible along the pathway. Here, we will concentrate on methods to study the structure of the folding intermediate state. 


\subsubsection{Experimental methods for studying folding intermediates}

While low-resolution spectroscopic techniques such as circular dichroism or tryptophan fluorescence can be used to probe the structure of the intermediate state[9] (they report on secondary structure formation and hydrophobic burial, respectively), an atomic-resolution picture provides much more information. Classically, experimentalists have tried to energetically trap the intermediate state such that it can be studied directly at the atomic resolution by solution nuclear magnetic resonance (NMR) spectroscopy. The difficulty to this approach lies in the design of appropriate mutations. Generally, native hydrogen/deuterium (H/D) exchange is used to identify regions which are disordered in the folding intermediate[7, 23-25]. These regions are then mutated under the assumption that the native state but not the folding intermediate will be destabilized, and the structure of the resulting intermediate mimic is solved by traditional nuclear Overhauser effect (NOE)-based NMR spectroscopy[10, 26-29]. However one must always consider the possibility that the "intermediate state" thus created is an artifact of mutation. Although the mutant can be shown to have similar folding kinetics to the intermediate, which provides a rough measure of their similarity, no high-resolution means of cross-validation exists.

The relatively new technique of relaxation dispersion NMR circumvents these issues as it can be used to solve the structure of the wild type intermediate state. Relaxation dispersion NMR, described in greater detail in the next section, can be used to obtain chemical shift values, residual dipolar couplings (RDCs) and chemical shift anisotropies (RCSAs) for the intermediate state (reviewed in [30]). These constitute powerful structural restraints that can be combined with a database structure prediction program such as CS-Rosetta in order to solve the structure of the wild type folding intermediate[1, 31, 32]. Furthermore, this method contains an intrinsic means of cross-validation. Similarly to the H/D exchange-based method described above, mutations that increase the intermediate state population can be designed based on the structure generated by relaxation dispersion, and the structure of the mutant can be solved by traditional NMR methods. Furthermore, the validity of the mutant as a mimic of the intermediate state can be assessed by comparing its chemical shifts to those of the wild type intermediate obtained by relaxation dispersion. A high level of confidence in the relaxation dispersion structure of the wild type intermediate can be obtained if it is shown to agree with that of an intermediate mimic with similar chemical shifts solved by traditional NMR methods. 


\subsubsection{CPMG relaxation dispersion NMR spectroscopy}

As described in section 1.1.2, high-energy folding intermediates continue to be populated at equilibrium even though the native state is dominant. Their population is so low, however, that they are "invisible" by solution NMR, which shows only the native state. Carr-PurcellMeiboom-Gill (CPMG) [33, 34] relaxation dispersion NMR spectroscopy can be used to tease out the "invisible" spectrum of a protein's high-energy excited state from the visible, ground state spectrum, as long as conditions can be found such that the intermediate is populated at $0.5 \%$ or higher and is exchanging with the ground state at a rate of a few hundred to several thousand per second[35]. A variable number of refocusing pulses is applied during a spin-echo pulse train, modulating the effective transverse relaxation rates of NMR active nuclei $\left({ }^{1} \mathrm{H},{ }^{13} \mathrm{C},{ }^{15} \mathrm{~N}\right)$ (reviewed in [36]). Fits of such rates as a function of the frequency of application of refocusing pulses to a model of chemical exchange leads to the extraction of the kinetic and thermodynamic parameters of the exchange process and of the chemical shift differences between interconverting states, $|\Delta \varpi|[21,35]$. For the case where the protein native state is in exchange with a transient intermediate state, the extracted parameters are $\mathrm{k}_{\mathrm{IN}}, \mathrm{k}_{\mathrm{N}}, \Delta \mathrm{G}_{\mathrm{IN}}$ and $\left|\Delta \varpi_{\mathrm{IN}}\right|$. Values of $|\Delta \varpi|$ can, in turn, be recast into shifts of the 'invisible' excited state once the sign of $\Delta \varpi$ is known from additional experiments[37-39]. Backbone ${ }^{15} \mathrm{~N}[40,41],{ }^{1} \mathrm{HN}[42],{ }^{13} \mathrm{C \alpha}[43],{ }^{13} \mathrm{CO}[44,45]$ and ${ }^{1} \mathrm{Ha}[46]$ chemical shifts of the excited state can now be measured using a variety of different CPMG relaxation dispersion experiments. In addition, similar experiments have been developed for quantifying the relative orientations of bond vectors in the excited state via measurement of residual dipolar couplings (RDCs) on samples that are fractionally aligned[47].

\subsubsection{Protein structure calculation by relaxation dispersion NMR}

Chemical shifts and RDC values provide a powerful set of structural restraints for studies of both ground and transiently formed excited states of proteins[1, 31, 48-51]. Chemical shifts in particular provide so much information that programs have been developed to generate protein structures using chemical shifts as the sole experimental restraint, although other restraints such as RDCs can be incorporated to improve structure quality[48-51]. Typically a protocol is used whereby small fragments are selected from a database of structures based on agreement with experimental data, with models derived subsequently from fragment assembly. The approach has 
been cross-validated for the native states of a large number of small to medium sized proteins (approximately 150 residues or less) for which high resolution structural information is available from either NMR or X-ray studies, and in the majority of cases the structures produced by the database approach are within $2 \AA$ of those generated using traditional methods[48, 49].

\subsubsection{Model proteins to study folding}

The techniques currently available to study protein folding are most easily applied to small, single domain proteins, often excised from larger proteins. They lack disulphide bonds and cofactors and can fold independently of chaperones. Furthermore, their small size ensures that they likely fold the same way in vitro as in vivo. Another advantage to their size is that it enables the extensive mutational studies required for $\varphi$-value analysis and makes them ideal candidates for NMR H/D exchange or relaxation-dispersion studies.

Several classes of model proteins have been studied to date, including all-beta sheet proteins such as the SH3 domain[21, 52], and all- $\alpha$-helical proteins such as the FF domain[1, 9, 53, 54]. Many of the all- $\alpha$-helical domains fold via a three-state mechanism, with a first rapid ( $\mu \mathrm{s})$ jump to a compact on-pathway intermediate state and subsequent slow (ms) rearrangement to the native conformation. Examples include the FF domain[9], Im7[8], the R16 and R17 domains of a-spectrin[55] and the engrailed homeodomain[7]. The folding intermediates of all of the aforementioned proteins form non-native tertiary interactions [1, 55-57], and in some cases, such as the engrailed homodomain and FF domain, have non-native helical boundaries. They fold via a framework or nucleation condensation mechanism[54, 56, 58-60], which are probably two manifestations of a single mechanism[61].

\subsection{FF domains}

FF domains are small, 4-helix bundles that bind to the phosphorylated C-terminal repeat domain (CTD) of the largest subunit of RNA polymerase II via a positively-charged cleft between the Ntermini of helices 1 and 4 [62].

\subsubsection{FF domains bind to the CTD of RNA polymerase II}

The CTD is a binding site for many proteins, most of which regulate mRNA processing and chromatin morphology (reviewed [63]). This unstructured domain contains multiple tandem 
repeats of the heptad sequence $\mathrm{Y}^{1} \mathrm{~S}^{2} \mathrm{P}^{3} \mathrm{~T}^{4} \mathrm{~S}^{5} \mathrm{P}^{6} \mathrm{~S}^{7}$ which can be phosphorylated at its $2^{\text {nd }}$ and $5^{\text {th }}$ serine positions[64]. The CTD remains unphosphorylated during transcription initiation, but becomes hyper-phosphorylated at the elongation phase[65, 66]. It is the pattern of phosphorylation during elongation that determines which set of factors can bind. For example, factors involved in pre-mRNA 5' capping are recruited at the beginning of elongation, when mainly $S^{5}$ is phosphorylated[67], and 3' cleavage and polyadenylation factors are recruited near the end of the gene, when only $S^{2}$ is phosphorylated[68]. Many other factors are recruited at some point during transcription, when both serines are phosphorylated, including mRNA splicing factors[69, 70] and histone acetyl transferases[71]. Binding to the phosphorylated CTD is mediated mainly by WW, SRI and FF domains, often found in tandem repeats that allow multiple binding interactions along the CTD.

\subsubsection{The FF domain from HYPA/FBP11 folds via an on-pathway intermediate}

The structure of an FF domain, that of the human splicing-related protein HYPA/FBP11, was solved in 2002 by Mark Bycroft and coworkers using solution NMR spectroscopy[62]. It is a 71 residue 4-helix bundle with a $\mathrm{H} 1(\alpha)-\mathrm{H} 2(\alpha)-\mathrm{H} 3\left(3_{10}\right)-\mathrm{H} 4(\alpha)$ topology (Figure 2, left-hand side).

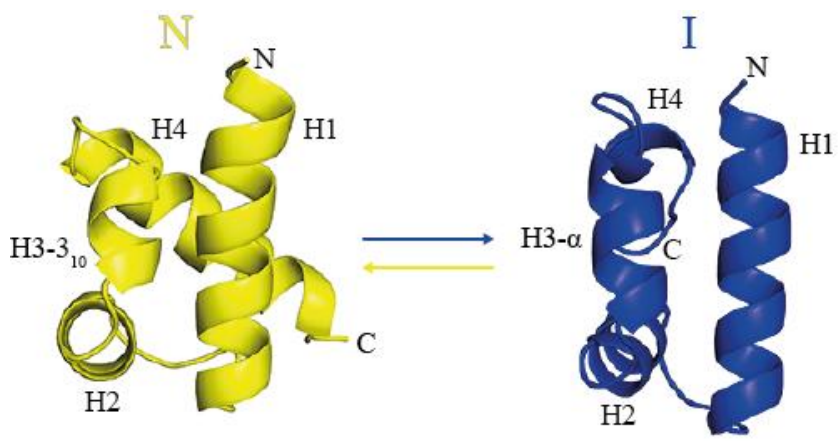

Figure 2. Structure and packing in the FF domain native state (N, left) and folding intermediate (I, right). The structure of $\mathrm{N}$ was solved by solution NMR spectroscopy[62], pdb accession code 1UZC, while I was solved by relaxation dispersion/CS-Rosetta[1], pdb accession code 2KZG. The topology of helices $\mathrm{H} 1$ and $\mathrm{H} 2$ is similar between the two states. H3 is elongated in the intermediate, with an $\alpha$-helix instead of $3_{10}$-helix topology, and forms multiple non-native interactions with $\mathrm{H} 1$ and $\mathrm{H} 2$. $\mathrm{H} 4$ has only partial helicity in the intermediate.

In 2004, it was found by Sir Alan Fersht and coworkers that the FF domain follows a three-state folding pathway involving a low-energy on-pathway intermediate[9]. Formation of the intermediate from the unfolded ensemble occurs quickly in a matter of microseconds, while rearrangement to the native state is slower, on the millisecond timescale. Kinetic modeling of the 
results of a combination of stopped-flow and continuous-flow experiments monitored by tryptophan fluorescence showed that the intermediate is on-pathway as the fluorescence traces fit better to a simulated on-pathway $(\mathrm{U} \leftrightarrow \mathrm{I} \leftrightarrow \mathrm{N})$ rather than off-pathway $(\mathrm{I} \leftrightarrow \mathrm{U} \leftrightarrow \mathrm{N})$ folding scheme. The rate-limiting transition state (between I and N) was later studied by Fersht and coworkers using $\varphi$-value analysis[54]. They found that native secondary and tertiary interactions are conserved in a single region of the transition state comprising the C-terminus of $\mathrm{H} 1$, the $\mathrm{H} 1-\mathrm{H} 2$ loop, and the $\mathrm{N}$-terminus of $\mathrm{H} 2$, which strongly suggests a nucleation-condensation mechanism of folding.

The kinetic intermediate was studied at equilibrium by Lewis Kay and coworkers by relaxation dispersion NMR[53]. At $25^{\circ} \mathrm{C}$, the FF intermediate is populated at around $2 \%$ and exchanges with the native state at a rate of about $2000 \mathrm{~s}^{-1}$. These properties are ideal for relaxation dispersion-based structural characterization (see section 1.2.2). The relaxation dispersion data for the wild type domain could best be modeled by a 2 -state $\mathrm{I} \leftrightarrow \mathrm{N}$ equilibrium, as the population of the unfolded state is undetectable $(<0.5 \%)$ at equilibrium. Two point mutants with an increased unfolded state equilibrium population were produced in order to assess whether the thermodynamic intermediate state is on- or off-pathway. The 3-state equilibrium data for the mutants was better modeled as on-pathway $(\mathrm{U} \leftrightarrow \mathrm{I} \leftrightarrow \mathrm{N})$ than off-pathway ( $\mathrm{I} \leftrightarrow \mathrm{U} \leftrightarrow \mathrm{N}$ or $\mathrm{U} \leftrightarrow \mathrm{N} \leftrightarrow \mathrm{I})$, in agreement with what had been found previously.

\subsubsection{Structure of the FF intermediate by relaxation dispersion NMR}

The structure of the folding intermediate was solved in 2010 by Kay and coworkers using CPMG relaxation dispersion NMR and CS-Rosetta modeling, as described in section 1.2.3[1]. Not surprisingly, the intermediate was found to share many aspects of structure with the native state (Figure 2, right-hand side). For example, the orientations and lengths of the first two helices H1, $\mathrm{H} 2$ and the intervening loop are similar in both states. However, there are distinct differences as well. In particular, H3 is $\alpha$-helical and significantly longer in the intermediate, consisting of residues that comprise both helix $\mathrm{H} 3$ and the $\mathrm{H} 3-\mathrm{H} 4$ loop in the native structure. The intermediate state structure is consistent with the previously reported kinetic and $\varphi$-value data: the lengthening of helix $\mathrm{H} 3$ generates a significant number of non-native interactions that must be broken prior to formation of the native structure, which is consistent with the observed slower 
second phase of folding, and the intermediate structure is only fully native-like in the H1-loop$\mathrm{H} 2$ region predicted by $\varphi$-value analysis.

A number of additional experiments performed at the time of the structure calculation suggested, but did not prove, that the proposed intermediate state structure was correct[1]. First, both the fold and the position of side-chains in the native state FF domain were reproduced using only backbone chemical shifts of the wild-type native state in concert with CS-Rosetta, providing confidence in the protocol used for calculations of I. Second, the structure of I was tested by making truncation mutations that were predicted to have little effect on I while drastically affecting the stability of the native conformer. One such truncation involves removal of the Cterminal residues, 61-71, that form $\alpha$-helix $\mathrm{H} 4$, since these residues are critically important for the $\mathrm{N}$ state, while they are partially disordered in I[1, 54], see Figure 2. The resultant mutant, $\mathrm{FF}_{1-60}$, folds with a single kinetic phase[1] and with a rate that is identical to that of the $\mathrm{U}$ to I transition in the wild-type protein[9], suggesting that $\mathrm{FF}_{1-60}$ is a mimic of I. Moreover, while ${ }^{1} \mathrm{HN}-{ }^{15} \mathrm{~N}$ correlation spectra of $\mathrm{FF}_{1-60}$ established the presence of two separate conformers in slow exchange, the chemical shifts of one set were very similar to those of I, confirming that indeed $\mathrm{H} 4$ is not critical to the formation of the intermediate state, as expected on the basis of the I state structure. Unfortunately, the poor chemical shift resolution, in particular for the sidechains, exacerbated by the extra set of correlations made further structural analysis on $\mathrm{FF}_{1-60}$ not possible.

\subsection{Rationale}

The relaxation dispersion NMR/data-base modeling protocol for solving the structure of folding intermediates provides an attractive alternative to the traditional method which requires mutagenic enhancement of the intermediate population. It also provides a method for studying other types of functional excited states, such as transiently populated catalytic[72-74] or ligandbinding conformations[75]. However, for this method to continue to be employed, one must be confident that the excited state structure is accurate. The traditional manner of solving protein structures by NMR spectroscopy is based on nuclear Overhauser effect spectroscopy (NOESY) inter-proton distance restraints. NOESY experiments exploit the phenomenon of cross-relaxation between hydrogen atoms that are close in space. Cross-peaks are generally visible between atoms that are separated by less than $6 \AA$, and their intensity is proportional to $1 / \mathrm{r}^{6}$, where $\mathrm{r}$ is the 
distance between protons. With enough NOE distance restraints it is possible to restrain the relative atomic positions in a protein. The CS-Rosetta calculation for the FF intermediate did not contain any such distance restraints, and there is therefore no direct evidence that the relative positions of atoms in the structure are correct.

In this thesis, we seek to validate the excited state structure of the FF domain intermediate by comparing it to a structure generated by an NOE-based approach. We have used the method of mutagenically enhancing the intermediate population by destabilizing the native state to produce an FF domain variant whose lowest energy conformation resembles that of the intermediate. We expand on the previous mutagenic work that produced $\mathrm{FF}_{1-60}$ from the wild type $\mathrm{FF}$ domain[1], and show that further truncation of the first ten residues generates a single, highly populated monomeric conformer $\left(\mathrm{FF}_{11-60}\right)$ that is a good mimic of the wild-type folding intermediate and can be studied in detail by solution NMR. We refer to this mimic as I', while the wild type intermediate studied by relaxation dispersion is I. We solved the structure of I' using traditional NOE based NMR methods. Good agreement between the structures of I' and I is obtained (the two structural ensembles are within $2 \AA$ of each-other), with the non-native interactions found in I reproduced in the I' model. These results provide strong validation of the previously reported folding intermediate structure, and provide strong evidence that relaxation dispersion NMR/CSRosetta is an accurate method for structural studies of invisible excited states. 


\section{Chapter 2 \\ Materials and Methods}

The FF $F_{11-60}$ plasmid was produced by Tomasz L. Religa. NMR experiments were performed with the help of Ranjith Muhandiram. The pulse sequence for recording Ala NOEs (Figure 4) was written by Lewis E. Kay.

\subsection{Sample production}

\subsubsection{Cloning of the $\mathrm{FF}_{11-60}$ plasmid}

$\mathrm{FF}_{11-60}$ was produced from a modified pRSET vector (Invitrogen) encoding $\mathrm{FF}_{1-71}$ using Quick Change PCR to delete residues 1-10 and 61-71 and to mutate residue 11 from $\mathrm{W}$ to $\mathrm{G}$. The resulting construct expresses an N-terminally His-tagged lipo domain separated from the following FF domain by a thrombin cleavage site[54]. An A51L mutant, used to disambiguate the Ala methyl region of the ${ }^{1} \mathrm{H}^{13} \mathrm{C}$ HSQC spectrum (discussed further in section 2.2.3), was likewise produced by Quick Change PCR from $\mathrm{FF}_{11-60}$.

\subsubsection{Protein expression}

BL21(DE3) Escherichia coli were transformed with plasmid DNA and starter cultures were grown overnight at $25^{\circ} \mathrm{C}$ in $100 \mathrm{ml} \mathrm{LB}$ media supplemented with $100 \mu \mathrm{g}$ ampicillin. Overnight LB cultures were pelleted and resuspended in 1L M9 minimal media to a starting OD600 of 0.1. Minimal media was supplemented with $100 \mathrm{mg}$ ampicillin as well as ${ }^{15} \mathrm{NH}_{4} \mathrm{Cl}$ and ${ }^{13} \mathrm{C}_{6}$-glucose as the sole nitrogen and carbon sources, respectively. For the stereospecific assignment of Leu $\delta$ and Val $\gamma$ methyl groups (discussed further in section 2.2.2), a mixture of $10 \%{ }^{13} \mathrm{C}_{6}$-glucose and $90 \%{ }^{12} \mathrm{C}_{6}$-glucose was added to the minimal media. M9 cultures were grown at $37^{\circ} \mathrm{C}$ for 5-7 hours until $\mathrm{OD} 600 \approx 0.7-0.8$ at which point temperature was decreased to $25^{\circ} \mathrm{C}$ and $1 \mathrm{mM}$ IPTG was added to induce overnight protein expression.

\subsubsection{Protein purification}

Following the established protocol for FF domain purification[54], the overnight cultures were pelleted and resuspended in lysis buffer $(50 \mathrm{mM}$ sodium phosphate, $20 \mathrm{mM}$ imidazole, $400 \mathrm{mM}$ 
$\mathrm{NACl}, \mathrm{pH}$ 8.0) with protease inhibitors, lysozyme and DNase I. Cells were lysed by sonication and the soluble fraction containing the FF domain was isolated by centrifugation. The supernatant was subjected to column Ni-affinity purification. The column was washed with 5-10 column volumes of lysis buffer and the protein was eluted with 3-4 column volumes of elution buffer (20mM sodium phosphate, $250 \mathrm{mM}$ imidazole, $\mathrm{pH} 8.0$ ). To remove the His-tagged lipo domain, approximately 50 units of bovine plasma thrombin (Amersham Biosciences) were added to the eluate which was then incubated at room temperature overnight. After 4-fold dilution with water to reduce the ionic strength of the buffer to around $5 \mathrm{mS} / \mathrm{cm}$, the digested sample was loaded onto a HiTrap SP column (Amersham Biosciences) equilibrated with 20mM Tris (pH8.0). A gradient of $0-0.5 \mathrm{M} \mathrm{NaCl}$ in $20 \mathrm{mM}$ Tris (pH8.0) was used to elute bound proteins. Fractions containing the FF domain were pooled and buffer exchanged into the NMR buffer $(20 \mathrm{mM}$ sodium acetate, $50 \mathrm{mM} \mathrm{NaCl}, \mathrm{pH} 4.8$ ). Prior to NMR measurements, $0.05 \%$ sodium azide and $5 \%$ $\mathrm{D}_{2} \mathrm{O}$ were added to the sample. Protein concentrations were between 1-2mM.

\subsection{NMR spectroscopy}

\subsubsection{Resonance assignments}

Backbone and side-chain resonance assignments were obtained using a set of gradient and sensitivity enhanced triple resonance experiment(HNCACB, CBCACONNH, CCC-TOCSY, HCC-TOCSY, HNCO, and HACACONNH)[76, 77], supplemented with a simultaneous ${ }^{15} \mathrm{~N}$-,

${ }^{13} \mathrm{C}$-edited NOESY-HSQC data set[78]. All experiments were performed at $25^{\circ} \mathrm{C}$ on a $500 \mathrm{MHz}$ spectrometer. The HNCACB and CBCACONNH experiments provided sequential assignments along the protein backbone. CCC-TOCSY provided side-chain carbon assignments, while HCCTOCSY and the ${ }^{15} \mathrm{~N}-,{ }^{13} \mathrm{C}$-edited NOESY provided side-chain proton assignments. The HNCO and HACACONNH experiments were used to assign $\mathrm{CO}$ and $\mathrm{H} \alpha$ chemical shifts, respectively. Complete backbone assignments were obtained for all residues except N-terminal residue G11, and residues N12, K28 and R29 which are broadened.

\subsubsection{Sterospecific Leu and Val methyl assignments}

Methyl groups are located predominantly in the protein hydrophobic core. As such, they provide a key source of highly restraining long-range NOEs. To maximize the number of unambiguous 
methyl distance restraints, it is important to uniquely assign each group. Stereospecific assignment of pro-R and pro-S Leu $\delta$ and Val $\gamma$ methyl groups was achieved using the method of Neri et al[79], in which one records a constant time ${ }^{1} \mathrm{H}-{ }^{13} \mathrm{C}$ HSQC spectrum of the methyl region on a $10 \%{ }^{13} \mathrm{C}_{6}$-glucose labeled sample. The bacterial metabolic pathway is such that the pro-R methyl carbon and directly bonded carbon $(\mathrm{C} \gamma 1, \mathrm{C} \beta$ in Val and $\mathrm{C} \delta 1, \mathrm{C} \gamma$ in Leu) derive from the same pyruvate molecule, while the pro-S methyl and the adjacent carbon originate from different pyruvates. As a consequence, with a $10: 1$ ratio of ${ }^{12} \mathrm{C}$ - to ${ }^{13} \mathrm{C}$-glucose, the probability of simultaneous incorporation of ${ }^{13} \mathrm{C}$ into the methyl carbon and adjacent carbon positions is $100 \%$ for pro-R groups but only $10 \%$ for pro-S. The effect of simultaneous incorporation is signal inversion during the constant time period in the ${ }^{1} \mathrm{H}^{-}{ }^{13} \mathrm{C}$ HSQC experiment due to scalar coupling between the two labeled carbons. Therefore, the pro-R peaks will be negative, while the pro-S peaks will appear positive (Figure 3). Phasing is achieved based on the Met methyl peaks, which have no adjacent Carbon atom.

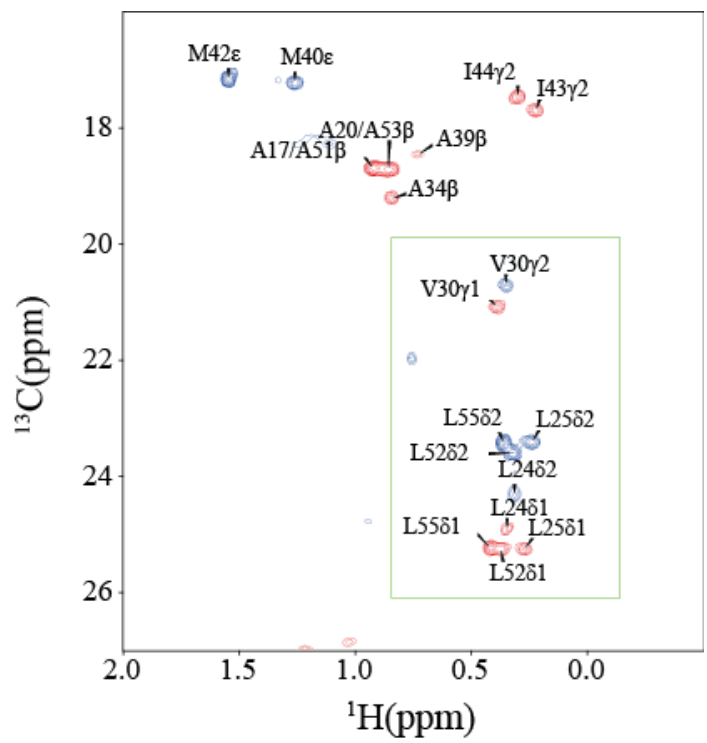

Figure 3. Stereospecific assignment of Leu $\delta$ and Val $\gamma$ methyl groups. A constant time ${ }^{1} \mathrm{H}-{ }^{13} \mathrm{C}$ HSQC spectrum of

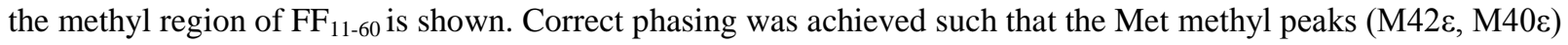
are positive (blue). The Val and Leu methyl region is found inside the green box. Pro-R methyl groups (Val $\gamma 1$, Leu $\delta 1$ ) show up as negative peaks (red), while pro-S methyls (Val $\gamma 2$, Leu $\delta 2$ ) are positive.

\subsubsection{NOESY distance restraints}

Inter-proton distance restraints were obtained from a simultaneous ${ }^{15} \mathrm{~N}$ - and ${ }^{13} \mathrm{C}$-edited NOESYHSQC data set[78] recorded with a mixing time of $150 \mathrm{~ms}(500 \mathrm{MHz})$. As the spectrum of $\mathrm{I}^{\prime}$ is 
characterized by poor chemical shift resolution, in particular for the side-chains, critical longrange methyl NOE restraints, including some involved in key non-native interactions, were obtained using higher resolution experiments, at a higher magnetic field. NOEs between methyl groups were obtained from a constant-time methyl-methyl NOESY experiment[80] (mixing time $=150 \mathrm{~ms}, 800 \mathrm{MHz}$ ). NOEs from Ala residues were detected using the pulse scheme illustrated in Figure 4 (mixing time of $250 \mathrm{~ms}, 800 \mathrm{MHz}$ ) whereby Ala $\left({ }^{1} \mathrm{H} \beta,{ }^{13} \mathrm{C} \beta\right)$ methyl chemical shifts were recorded in $\left(\mathrm{t}_{1}, \mathrm{t}_{2}\right)$, with the chemical shifts of proximal protons measured in the direct dimension.

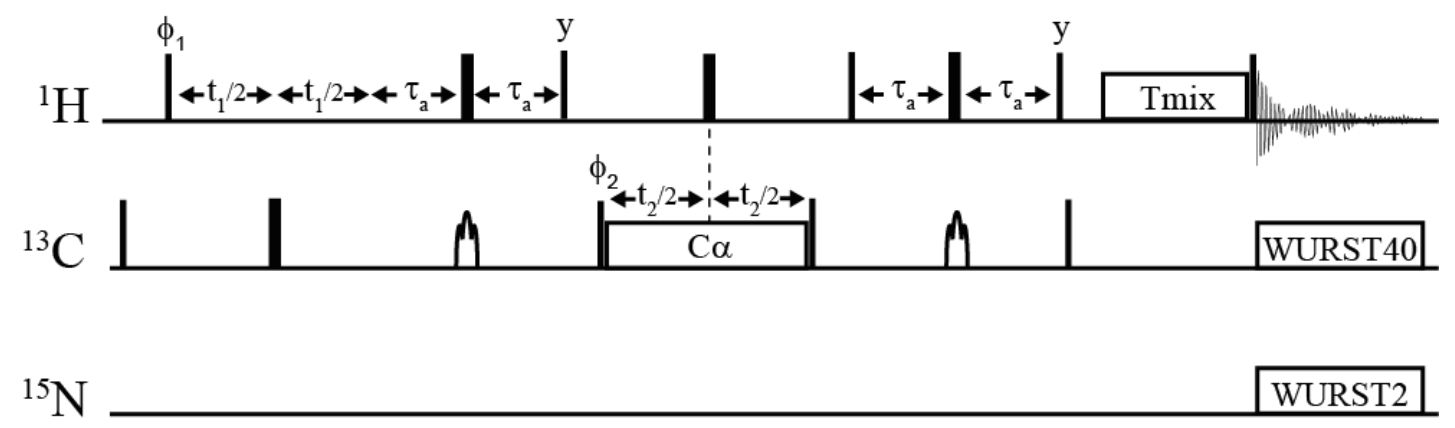

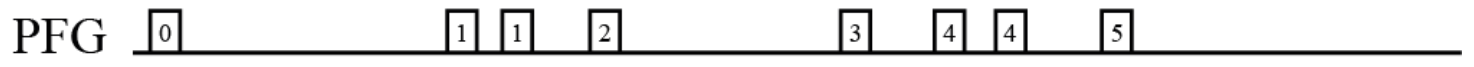

Figure 4. Pulse scheme for measurement of NOEs from Ala residues. Correlations of the form $\left(\varpi^{1} \mathrm{H}_{\mathrm{ALA}}, \omega^{13} \mathrm{C}_{\mathrm{ALA}}, \varpi^{1} \mathrm{H}\right)$ are detected where $\varpi^{1} \mathrm{H}_{\mathrm{ALA}}$ and $\varpi^{13} \mathrm{C}_{\mathrm{ALA}}$ are Ala methyl chemical shifts and $\varpi^{1} \mathrm{H}$ is the chemical shift of a proximal proton. All narrow (wide) rectangular pulses are applied with $90^{\circ}\left(180^{\circ}\right)$ flip angles along the $\mathrm{x}$ axis, unless indicated otherwise, at the highest possible power levels. ${ }^{1} \mathrm{H}$ and ${ }^{13} \mathrm{C}$ radio frequency carriers are positioned at water and $19 \mathrm{ppm}$, respectively, until signal acquisition when the carbon carrier jumps to $75 \mathrm{ppm}$. During the mixing time a $30 \mathrm{~Hz}{ }^{1} \mathrm{H}$ saturation field is applied to the water line. Shaped ${ }^{13} \mathrm{C}$ pulses $(2 \mathrm{~ms}$, Re-Burp profile[81], centered at $21.5 \mathrm{ppm}, 800 \mathrm{MHz}$ ) are selective to excite primarily ${ }^{13} \mathrm{C} \beta$ of Ala (along with other methyl spins). ${ }^{13} \mathrm{C} \alpha$ decoupling during $\mathrm{t}_{1}$ is achieved using constant adiabaticity WURST-8 decoupling[82], sweeping from 50 to $58 \mathrm{ppm}$. A second field is applied, swept from -12 to $-20 \mathrm{ppm}$, to reduce Bloch-Siegert effects for the Ala methyl ${ }^{13} \mathrm{C}$ spins[83]. The net decoupling field strength is $0.59 \mathrm{kHz}(\mathrm{rms} 0.33 \mathrm{kHz}) .{ }^{13} \mathrm{C}$ broad-band decoupling is achieved with a $2.6 \mathrm{kHz}$ (max rf; $2.3 \mathrm{kHz}$ rms) WURST-40 field centered at $75 \mathrm{ppm}$ [82]. ${ }^{15} \mathrm{~N}$ decoupling during acquisition was carried out using a WURST-2 field[82], with a bandwidth of $27 \mathrm{ppm}$, centered at $116.5 \mathrm{ppm}(0.75$ $\mathrm{kHz}$ max rf; $0.46 \mathrm{kHz}$ rms). The value of $\tau_{\alpha}$ is set to $2 \mathrm{~ms}$. The phase cycle is $\mathrm{f}_{1}=\mathrm{x},-\mathrm{x} ; \mathrm{f}_{2}=2(\mathrm{x}), 2(-\mathrm{x}) ;$ rec $=\mathrm{x}, 2(-\mathrm{x}), \mathrm{x}$. Quadrature in $\mathrm{F}_{1}$ is achieved by States-TPPI[84] of $\mathrm{f}_{2}$. The gradient durations (ms) and strengths $(\mathrm{G} / \mathrm{cm}$, along Z) are: $\mathrm{g} 0=(1,10), \mathrm{g} 1=(0.3,20), \mathrm{g} 2=(1,24), \mathrm{g} 3=(1.4,-40), \mathrm{g} 4=(0.5,-24), \mathrm{g} 5=(0.8,20)$.

The Ala methyl region of the ${ }^{1} \mathrm{H}^{-13} \mathrm{C}$ HSQC spectrum is characterized by a great deal of overlap. A53/A20 are overlapped, as are A17/A51, making it difficult to unambiguously assign a number of potential non-native NOE contacts. Therefore, the methyl-methyl NOESY and Ala NOESY experiments were performed on an A51L mutant. Mutation of A51, which is surface exposed, to Leu eliminated the A17/A51 ambiguity as well as the degeneracy of A53/A20 (Figure 5). The 
resulting ${ }^{1} \mathrm{HN}-{ }^{15} \mathrm{~N}$ 'fingerprint' spectrum showed essentially no changes, with only minor perturbations in the region of the mutation.
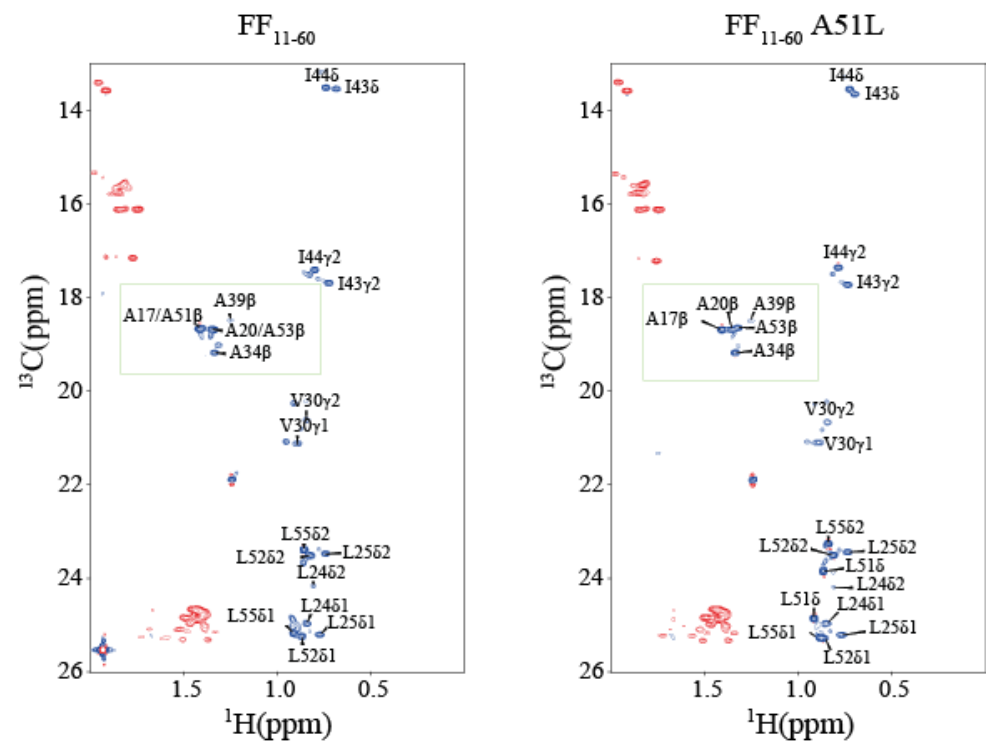

Figure 5. Effect of the A51L mutation on the methyl region of the ${ }^{1} \mathrm{H}-{ }^{13} \mathrm{C}$ HSQC spectrum. The Ala methyl region is highly overlapped for $\mathrm{FF}_{11-60}$ (inside green box, left). Mutation of A51, which is not involved in any critical interactions, to a Leu results in removal of ambiguity in the Ala region (inside green box, right) while the remainder of the spectrum (as well as the ${ }^{1} \mathrm{HN}-{ }^{15} \mathrm{~N}$ ' fingerprint' spectrum) remains virtually unchanged.

\subsection{Structure calculations}

\subsubsection{TALOS $+\varphi / \psi$ dihedral angle prediction}

The secondary structure of I' was initially assessed using the TALOS+ program[85], based on ${ }^{15} \mathrm{~N},{ }^{1} \mathrm{HN},{ }^{13} \mathrm{C} \alpha,{ }^{13} \mathrm{C} \beta,{ }^{13} \mathrm{CO}$ and ${ }^{1} \mathrm{H} \alpha$ chemical shifts. The program subtracts the residue-specific random coil shift from the experimental shift, generating a secondary chemical shift lacking residue-type-specific contributions. TALOS+ searches a database containing proteins from the biological magnetic resonance data bank (BMRB) as well as PDB coordinates from highresolution X-ray structures for the 10 best matches to the secondary shifts of each residue and its flanking neighbors. It uses the consensus $\varphi$ and $\psi$ angles from these 10 matches to predict those for the residue. From the magnitude and sign of the secondary shift of each residue and its flanking neighbors, TALOS+ also estimates the secondary structure propensity of that amino acid: an $\alpha$-helical conformation causes $\mathrm{C} \alpha$ and $\mathrm{CO}$ resonances to be shifted downfield from their 
random coil value while $\mathrm{H} \alpha$ and $\mathrm{C} \beta$ resonances are shifted upfield, towards lower chemical shifts. The opposite is true for a $\beta$-sheet conformation.

\subsection{2. $\mathrm{RCl}$ order parameter prediction}

The dynamics of I' were assessed using the built-in RCI (random coil index)[86] order parameter prediction module in TALOS+. Just as TALOS+ exploits the torsion angle information intrinsic to chemical shifts, the RCI method exploits their intrinsic dynamic information. The program first calculates a RCI value for each residue from its ${ }^{15} \mathrm{~N},{ }^{1} \mathrm{HN},{ }^{13} \mathrm{C} \alpha,{ }^{13} \mathrm{C} \beta,{ }^{13} \mathrm{CO}$ and ${ }^{1} \mathrm{H} \alpha$ secondary shifts using the expression

$\mathrm{RCI}=\left(<\mathrm{A}\left|\Delta \delta_{\mathrm{C \alpha}}\right|+\mathrm{B}\left|\Delta \delta_{\mathrm{CO}}\right|+\mathrm{C}\left|\Delta \delta_{\mathrm{C} \beta}\right|+\mathrm{D}\left|\Delta \delta_{\mathrm{N}}\right|+\mathrm{E}\left|\Delta \delta_{\mathrm{HN}}\right|+\mathrm{F}\left|\Delta \delta_{\mathrm{H \alpha}}\right|>\right)^{-1}$,

which is the inverse of an empirically-weighted sum of the absolute values of the various secondary shifts. RCI then uses the empirical function

$\mathrm{S}^{2}=1-0.5 * \ln (1+\mathrm{RCI} * 10.0)$

to correlate the calculated RCI value to a squared order parameter $\left(\mathrm{S}^{2}\right)$, which is a measure of protein dynamics. $S^{2}$ ranges between 0 and 1 , and the lower its value, the more dynamic the residue.

\subsubsection{XPLOR-NIH structure calculation}

XPLOR-NIH[87, 88] calculates protein structures based on NMR restraints by seeking the minimum of a target function comprising terms for the experimental restraints, covalent geometry and non-bonded contacts using rounds of molecular dynamics and Monte Carlo and gradient-based minimization. Here, experimental restraints included $58 \varphi$ and $\psi$ torsion angles for regions which TALOS+ predicted to be helical, as well as 121 NOE distance restraints: 37 sequential NOE $(|i-j|=1)$ constraints, 57 medium range NOE $(1<|i-j|<5)$ constraints and 27 long range NOE (all other) constraints. These were input into XPLOR-NIH using the script xplor-nih-2.27/eginput/protG/anneal.inp, modified to include the rama potential[89] for the ordered portion of the protein as predicted by TALOS+ (residues 15-54). The final force constants for distance, torsion angle restraints and Ramachandran database potential of mean force were $30 \mathrm{kcal} \mathrm{mol}^{-1}, 200 \mathrm{kcal} \mathrm{mol}^{-1}$, and $1.0 \mathrm{kcal} \mathrm{mol}^{-1}$, respectively. 100 trial structures 
were calculated and the ensemble of 10 best structures with the lowest NOE and dihedral angle violations were chosen to represent the final structures (no NOE violation greater than $0.5 \AA$, no dihedral angle violation greater than $5^{\circ}$ ). PROCHECK software[90] was used to analyze the Ramachandran plots. PROCHECK calculates the $\varphi$ and $\psi$ angles for each residue from the PDB files generated by XPLOR-NIH (or any other means) and analyses their distribution with regard to the different regions of the Ramachandran plot. It then calculates the percentage of residues in each of the most favored, additional allowed, generously allowed and disallowed regions of the plot. Standard statistics used to measure the quality of NMR structures are provided in Figure 6.

\begin{tabular}{|ll|}
\hline $\begin{array}{ll}\text { Restraints used for structure calculations } \\
\text { NOE distances }(\AA)\end{array}$ & 121 \\
Dihedral angles $\left(^{\circ}\right)$ & 58 \\
& \\
Average RMSD from idealized covalent geometry & \\
\hline Bond $(\AA)$ & $0.0016 \pm 0.0002$ \\
NOE $(\AA)$ & $0.033 \pm 0.012$ \\
Bond angles $\left(^{\circ}\right)$ & $0.376 \pm 0.004$ \\
Improper $\left(^{\circ}\right)$ & $0.323 \pm 0.068$ \\
& \\
Average RMSD within the structural ensemble & \\
Backbone & $0.79 \pm 0.23$ \\
Heavy atoms & $1.22 \pm 0.25$ \\
& \\
PROCHECK[90] Ramachandran analysis & $85.0 \%$ \\
Residues in most favored regions & $12.5 \%$ \\
Residues in additionally allowed regions & $2.5 \%$ \\
Residues in generously allowed regions & $0.0 \%$ \\
Residues in disallowed regions & \\
\hline
\end{tabular}

Figure 6. Structural statistics on the 10 lowest energy structures with no violations out of 100 calculated for I' using XPLOR-NIH[87, 88]. 


\section{Chapter 3 \\ Results and Discussion}

All work on $F_{1-60}$ was performed by Dmitry M. Korzhnev.

\subsection{Searching for an I state mimic as a quantitative test of structure}

The goal of the present work is to cross-validate the structure of the wild-type FF domain folding intermediate that has been solved on the basis of CPMG relaxation dispersion derived constraints and a CS-Rosetta structure determination protocol[1]. In an effort to produce an intermediate mimic with more favorable spectroscopic properties than $\mathrm{FF}_{1-60}$ (see section 1.3.4), we compared backbone chemical shifts of both sets of correlations from the pair of slowly exchanging $\mathrm{FF}_{1-60}$ conformers. We were able to ascertain that the major differences were in the N-terminus and that, further, the relaxation properties of one of the conformers were consistent with a dimeric structure. With this in mind, a second deletion was introduced in which the first ten residues were removed and a $\mathrm{W} 11 \mathrm{G}$ mutation added, to produce $\mathrm{FF}_{11-60}$. As discussed in more detail in the next section, ${ }^{1} \mathrm{HN}-{ }^{15} \mathrm{~N}$ HSQC spectra of $\mathrm{FF}_{11-60}$ were recorded showing a predominant single set of correlations, suggesting that this double truncated domain could form the basis for further structural studies.

The approach that we have taken to produce a potential I state mimic is schematized in Figure 7. The energy landscape of the wild-type FF domain is shown on the left-hand side with the relative stabilities of each of the states indicated (U, I and $\mathrm{N} ; 25^{\circ} \mathrm{C}$ ), established by stopped-flow fluorescence[9] and relaxation dispersion NMR[53]. Both the $\mathrm{N}$ - and C-termini have been cleaved to produce the landscape shown on the right, assuming little change in relative stabilities of $\mathrm{U}$ and $\mathrm{I}$. The $\mathrm{N}$ state can no longer form, since crucial contacts involving $\mathrm{H} 4-\mathrm{H} 1$ and $\mathrm{H} 4-\mathrm{H} 3$ are absent. Thus, the predominant state in solution, referred to as $\mathrm{I}^{\prime}$, can be studied using conventional NMR methods. We show in the next section that $\mathrm{I}^{\prime}$ is a good mimic of the I state. 


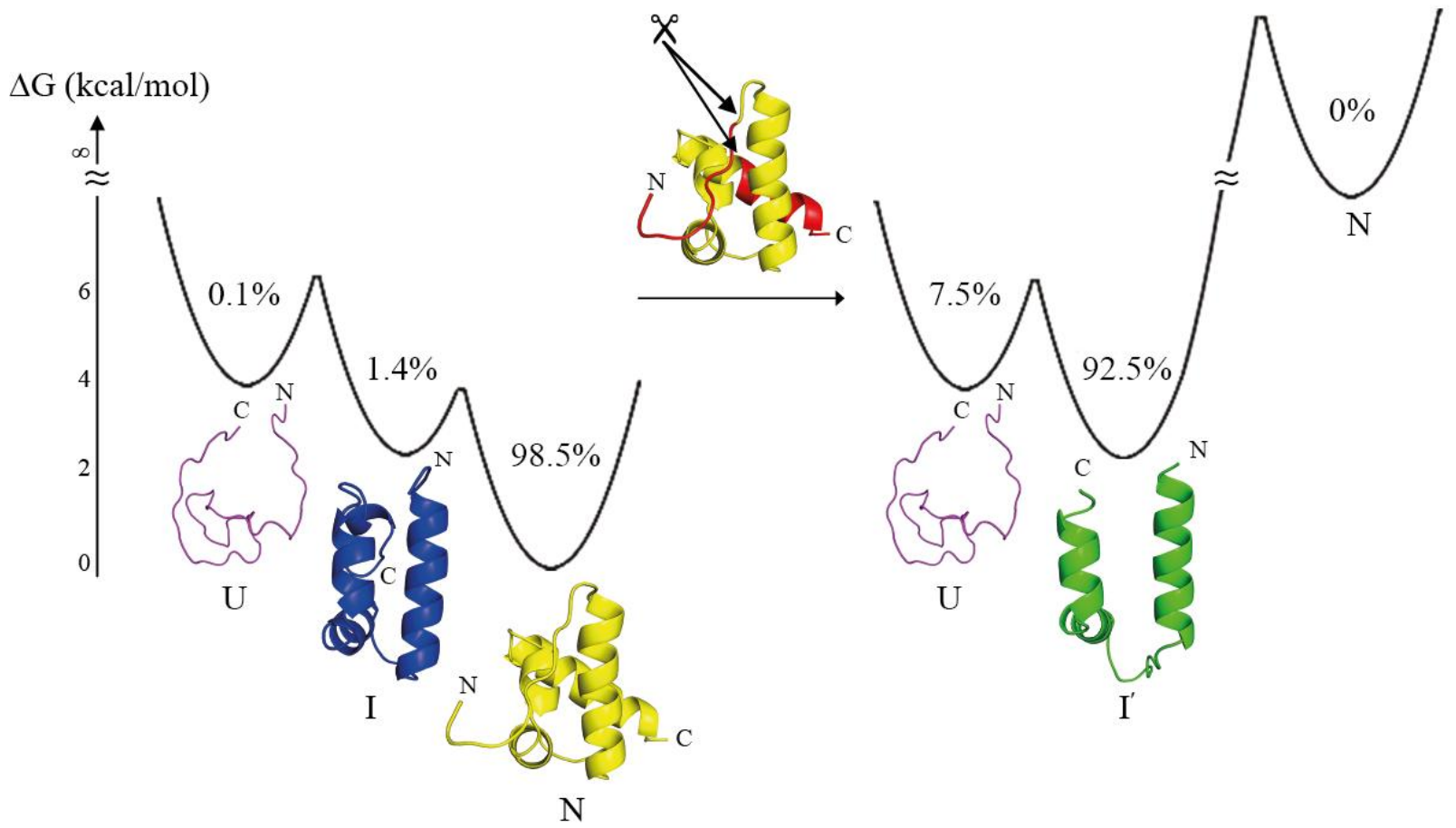

Figure 7. Populations and relative free energies of the unfolded (U), intermediate (I) and native states $(\mathrm{N})$ of $\mathrm{FF}_{1-71}$ (left), as determined by stopped-flow fluorescence[9] and relaxation dispersion NMR studies, $25^{\circ} \mathrm{C}[53]$. The structure of the $\mathrm{U}$ state (purple) is a schematic based on a molecular dynamics simulation in the absence of restraints, while the structures of I (blue) and $\mathrm{N}$ (yellow) indicated by the ribbon diagrams, where determined by relaxation dispersion NMR/CS-Rosetta[1] (I, pdb accession code 2KZG) and standard NMR[62] (N, pdb accession code 1UZC) approaches, respectively. Truncation of N- (1-10) and C-terminal (61-71) residues (indicated in red on the structure above the arrow) creates $\mathrm{FF}_{11-60}$ (right) with a dominant population of $\mathrm{I}^{\prime}$ (whose structure determined in the present study is indicated in green). We have assumed that the relative energies between states $\mathrm{I} / \mathrm{U}$ and $\mathrm{I}^{\prime} / \mathrm{U}$ are the same.

\subsection{FF11-60 is a good mimic of the FF folding intermediate}

Prior to detailed studies of $\mathrm{I}^{\prime}$ it is first important to establish that this molecule is a good mimic of I. The most straightforward and rapid way of 'screening' candidate molecules as good mimics is through a comparison of backbone chemical shifts with those of I. Figure 8 a shows a ${ }^{1} \mathrm{HN}-{ }^{15} \mathrm{~N}$ HSQC correlation map of I', with assignments of many of the cross-peaks indicated. Correlations for residue N12 located at the N-terminus of the protein, as well as for K28 and R29 at the Nterminus of the loop between $\mathrm{H} 1$ and $\mathrm{H} 2$, are not observed, however all other backbone positions could be assigned. In addition to the intense correlations shown in the Figure there are much weaker peaks for which assignments are not available. Figure $8 \mathrm{~b}$ compares ${ }^{15} \mathrm{~N},{ }^{1} \mathrm{HN},{ }^{13} \mathrm{C} \alpha,{ }^{1} \mathrm{H} \alpha$ and ${ }^{13} \mathrm{CO}$ chemical shift differences between $\mathrm{I}^{\prime}$ and the native state of $\mathrm{FF}_{1-71}\left(\Delta \omega_{\text {spec }}\right.$, Y-axis $)$ with chemical shift differences between the I and $\mathrm{N}$ states of $\mathrm{FF}_{1-71}$, as obtained previously from 
analysis of relaxation dispersion data-sets[1] $\left(\Delta \varpi_{\text {disp }}, X\right.$-axis $)$. The line $\Delta \varpi_{\text {spec }}=\Delta \varpi_{\text {disp }}$ is included in the figure as a guide.

(a)

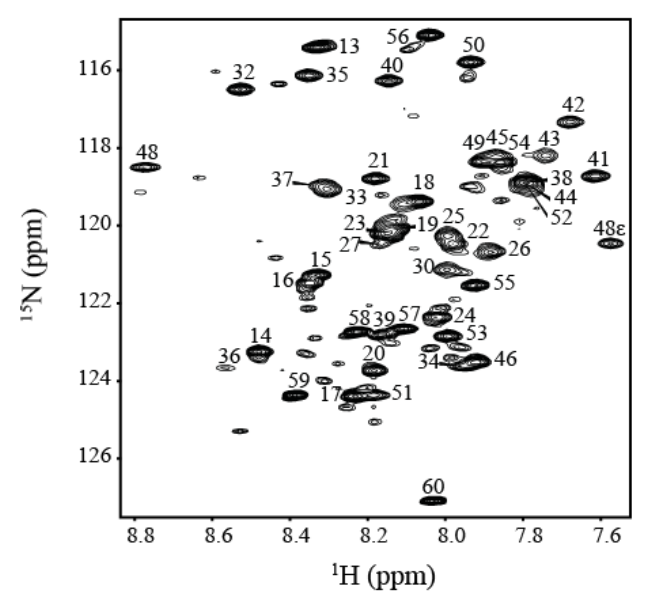

(c)
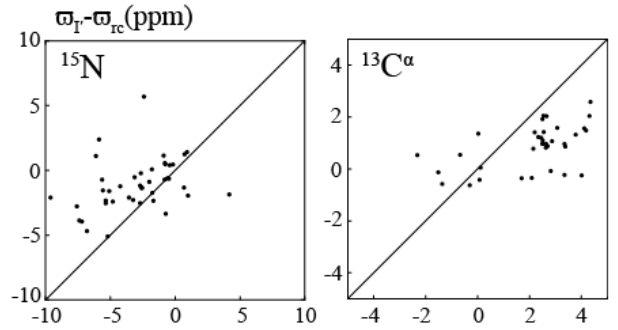

(b)
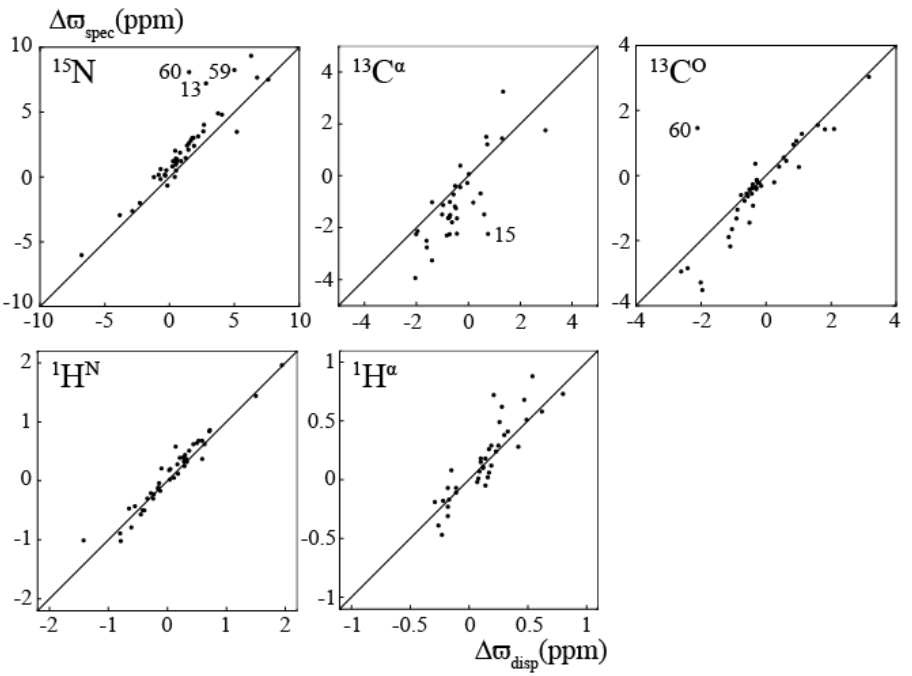
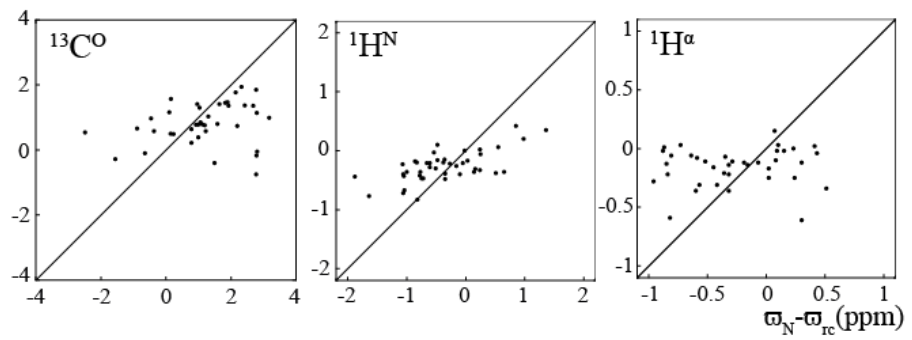

Figure 8. The I' state of $\mathrm{FF}_{11-60}$ is a good mimic of the $\mathrm{FF}_{1-71}$ folding intermediate. (a) ${ }^{1} \mathrm{HN}-{ }^{15} \mathrm{~N}$ HSQC correlation map of $\mathrm{FF}_{11-60}$, with chemical shift assignments as indicated. A number of weak peaks are present that are unassigned. (b) Linear correlation plots of chemical shift differences between I' and the $\mathrm{FF}_{1-71}$ native state $\left(\Delta \varpi_{\text {spec }}\right.$, $\mathrm{Y}$-axis) $v s$ the corresponding differences between $\mathrm{FF}_{1-71} \mathrm{I}$ and $\mathrm{N}$ states $\left(\Delta \varpi_{\text {disp }}, \mathrm{X}\right.$-axis $)$ for backbone ${ }^{15} \mathrm{~N},{ }^{1} \mathrm{HN},{ }^{13} \mathrm{C} \alpha$, ${ }^{1} \mathrm{H} \alpha$ and ${ }^{13} \mathrm{CO}$ spins. The $\mathrm{FF}_{1-71} \mathrm{I}$ and $\mathrm{N}$ chemical shifts have been published previously[1], while the chemical shifts of I' were obtained in the present study. The line $\Delta \omega_{\text {spec }}=\Delta \omega_{\text {disp }}$ is shown to guide the eye. RMSD values between datasets have been calculated, after correcting for the offset (i.e., non-zero $\left(\left\langle\Delta \omega_{\text {spec }}-\Delta \omega_{\text {disp }}\right\rangle\right.$ values), excluding outliers to the best fit line $\mathrm{y}=\mathrm{x}+\mathrm{b}$; values of $0.69,0.85,0.44,0.14$ and $0.18 \mathrm{ppm}$ are obtained for ${ }^{15} \mathrm{~N},{ }^{13} \mathrm{C} \alpha,{ }^{13} \mathrm{CO}$, ${ }^{1} \mathrm{HN}$ and ${ }^{1} \mathrm{H} \alpha$ spins, respectively. (c) Linear correlation plots of chemical shift differences between $\mathrm{I}^{\prime}$ and random coil shifts for the WT FF domain $\left(\varpi_{\mathrm{I}^{\prime}}-\varpi_{\mathrm{rc}}\right.$, y-axis) $v s$ shift differences between $\mathrm{FF}_{1-71}$ native and random coil states $\left(\varpi_{\mathrm{N}}-\varpi_{\mathrm{rc}}, \mathrm{X}\right.$-axis) for backbone ${ }^{15} \mathrm{~N},{ }^{1} \mathrm{HN},{ }^{13} \mathrm{C} \alpha,{ }^{1} \mathrm{H} \alpha$ and ${ }^{13} \mathrm{CO}$ spins. $\mathrm{FF}_{1-71}$ random coil and native state chemical shifts were obtained from previously published values[1].

It is clear that while there is a reasonably good correlation of chemical shifts, there is an offset

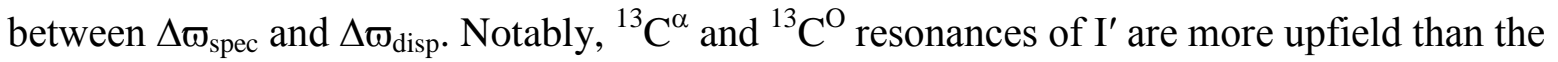
corresponding peak positions in I $\left(\left\langle\Delta \varpi_{\text {spec }}-\Delta \varpi_{\text {disp }}\right\rangle_{\mathrm{C} \alpha}=-0.59 \mathrm{ppm},\left\langle\Delta \varpi_{\text {spec }}-\Delta \varpi_{\text {disp }}\right\rangle_{\mathrm{CO}}=-0.24\right.$ ppm, where the angular brackets indicate averaging over all residues), while ${ }^{1} \mathrm{H} \alpha$ resonances of $\mathrm{I}^{\prime}$ are more downfield $\left.\left(<\Delta \varpi_{\text {spec }}-\Delta \varpi_{\text {disp }}\right\rangle_{\mathrm{H} \alpha}=0.05 \mathrm{ppm}\right)$, on average. These nuclei are sensitive reporters of secondary structure in proteins $[91,92]$ and the correlation between $\Delta \varpi_{\text {spec }}$ and $\Delta \varpi_{\text {disp }}$ 
is therefore consistent with $\mathrm{I}^{\prime}$ and I possessing similar secondary structures. The signs of $<\Delta \varpi_{\text {spec }}$ - $\Delta \varpi_{\text {disp }}>_{X}$, (and indeed also for ${ }^{15} \mathrm{~N}$ and ${ }^{1} \mathrm{H}^{\mathrm{N}}$, although these shifts are less diagnostic of secondary structure) suggests strongly that the extent of $\alpha$-helix secondary structure formation is less in the I' state. A more detailed analysis, given below, establishes that the positions of the helices are very similar in I' and I so that that the doubly-truncated variant is a good model of the wild-type FF domain folding intermediate that can be used to 'test' the structure of I determined by CS-Rosetta. It is worth pointing out that, not surprisingly, the chemical shifts of I' show no correlation with those of the $\mathrm{N}$ state of $\mathrm{FF}_{1-71}$ (Figure 8c).

\subsection{I and I' have similar secondary structures, but I' is more dynamic}

The backbone and ${ }^{13} \mathrm{C} \beta$ chemical shifts of I' have been input into the TALOS+ program[85] to predict residue-specific helical propensities. Not surprisingly, I' consists of 3 helices, H1-H3. The percent helicity $v s$. residue profile for I' is compared with that for I obtained in a similar manner in Figure 9a.

(a)

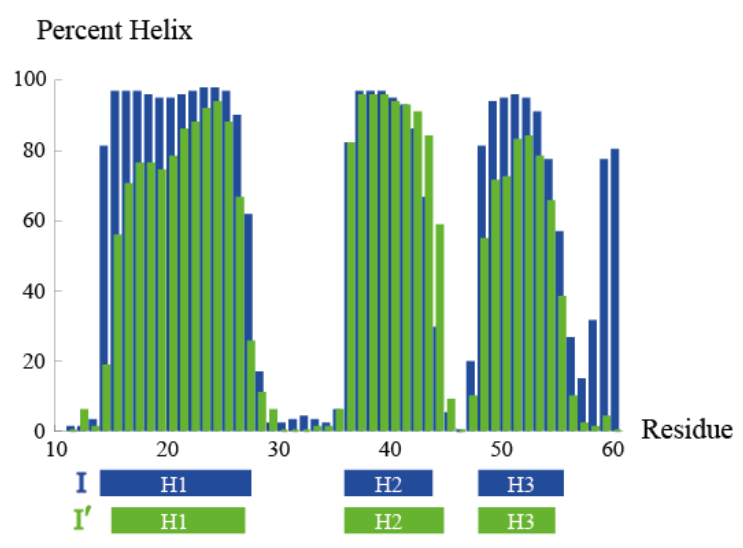

(b)

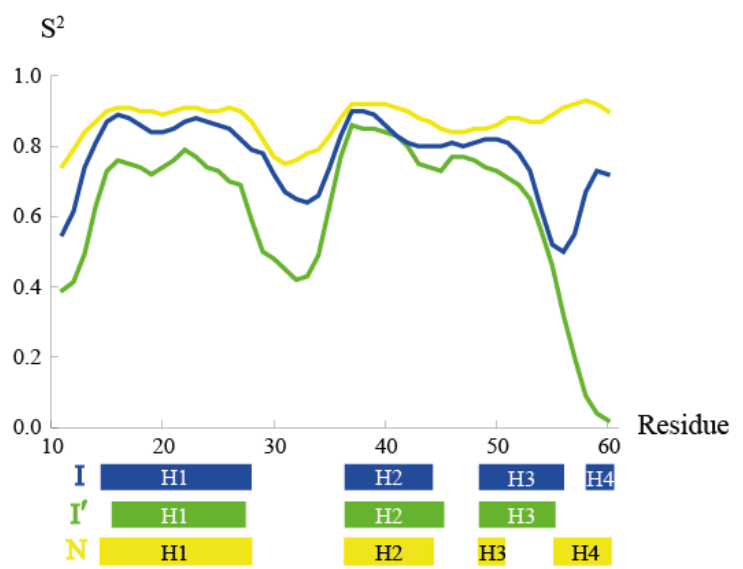

Figure 9. Comparison of per-residue helical propensities and backbone dynamics of I' (green) with those of the $\mathrm{FF}_{1}$ ${ }_{71}$ folding intermediate, I (blue). (a) TALOS+-predicted helix propensities [85] based on backbone chemical shifts for I and backbone and ${ }^{13} \mathrm{C} \beta$ shifts for $\mathrm{I}^{\prime}$. TALOS+ predicted helices are shown as rectangles and span residues 1427, 36-43 and 48-55 for I and 15-26, 36-44 and 48-54 for I'. (b) RCI[86]-predicted $\mathrm{S}^{2}$ values for the backbone amide groups of $\mathrm{I}^{\prime}, \mathrm{I}$ and $\mathrm{N}$.

Helices $\mathrm{H} 1$ and $\mathrm{H} 3$, that are adjacent to the truncation points, have lower helical propensity in I'. This most likely reflects partial destabilization of these elements caused by loss of adjacent residues. By contrast, the helical content of $\mathrm{H} 2$ is very similar in I' and I. Notably, very similar helix boundaries are predicted for the two states, with $\mathrm{H} 1, \mathrm{H} 2$ and $\mathrm{H} 3$ spanning residues 15-26, 
36-44 and 48-54 in I' compared to 14-27, 36-43 and 48-55 for I. The flexibility of the amides in I' and I have been calculated from backbone chemical shifts using the RCI approach[86] and plotted as squared order parameters $\left(\mathrm{S}^{2}\right)$ in Figure $9 \mathrm{~b}$. Helices $\mathrm{H} 1$ and $\mathrm{H} 3$ are more dynamic in $\mathrm{I}^{\prime}$, with $\mathrm{S}^{2}$ values between 0.7-0.8 (H1), 0.75-0.85 (H2) and 0.7-0.75 (N-terminus of H3) compared to 0.8 -0.9 for the helices of I, with order parameters dropping off at the $\mathrm{C}$-terminus of $\mathrm{H} 3$ for both variants. By means of comparison, we have also plotted RCI based $\mathrm{S}^{2}$ values for the N state of the wild-type FF domain[1]. The increased dynamics of I' relative to what is observed in the native state in part once again reflects rapid averaging between $\mathrm{I}^{\prime}$ and $\mathrm{U}$ (that is populated close to $10 \%$ in $\mathrm{FF}_{11-60}$, Figure 7), leading both to a decrease in spectral resolution and some peak broadening relative to data sets recorded of the wild-type protein.

\subsection{I' forms non-native contacts predicted by the CS-Rosetta structure of I}

A key difference in the structures of the $\mathrm{N}$ and I states of the wild-type FF domain is that a series of non-native interactions are formed in I as a consequence of the elongated helix $\mathrm{H} 3$ in the intermediate[1], Figure 10a. For example, the Y49-L55 contact in the $\mathrm{N}$ state is replace by a Y49-A53 interaction in I (cyan), L55 in the I state is proximal to A17 (red) and residues L52A20 (green) are closer in the intermediate then in the native structure. The distances between these amino acid pairs in both the $\mathrm{N}$ and I conformations are given in Figure 10a. The fact that $\mathrm{I}^{\prime}$ serves as a good mimic of the intermediate state provides an opportunity to rigorously test the CS-Rosetta structure of I by independently verifying the presence of these non-native contacts. In principle for a small domain of 50 residues this should be easily accomplished by analysis of a ${ }^{13} \mathrm{C}$-edited 3D NOESY data set. However, it is clear that $\mathrm{I}^{\prime}$ is much more dynamic than a 'typical' native protein, as might be expected for a domain that is only $1.5 \mathrm{kcal} / \mathrm{mol}$ more stable than its unfolded state (Figure 7). Decrease in spectral resolution in key regions of the ${ }^{1} \mathrm{H}-{ }^{13} \mathrm{C}$ correlation map makes it difficult to unambiguously assign a number of the potential non-native NOE contacts, however the ambiguities can be lifted by an A51L point mutation (see section 2.2.3). Figure $10 \mathrm{~b}$ shows a number of strip-plots from a methyl- ${ }^{13} \mathrm{C}$-edited NOESY data set linking proximal methyl groups. Here ${ }^{13} \mathrm{C}$ chemical shifts for both the origination and destination methyl were recorded in constant-time mode so that a high resolution map could be obtained[80]. A second data set was recorded in which Ala $\left({ }^{1} \mathrm{H} \beta,{ }^{13} \mathrm{C} \beta\right)$ methyl chemical shifts were recorded in $\left(\mathrm{t}_{1}, \mathrm{t}_{2}\right)$, with the chemical shifts of proximal protons measured in the direct 
dimension, Figure 10c (refer to Figure 4 for pulse sequence details). Notably, NOE correlations are observed between A17H $\beta / \mathrm{L} 55 \mathrm{H} \delta$, A20H $\beta / \mathrm{L} 52 \mathrm{H} \delta$ (Figure 10b) and between A20H $\beta / \mathrm{Y} 49 \mathrm{H} \varepsilon$ (Figure 10c) that are all predicted from the structure of the wild-type intermediate, while none of these contacts were noted in studies of the native state FF domain[62], as expected (BMRB and PDB accession codes 5537 and 1UZC).

(a)

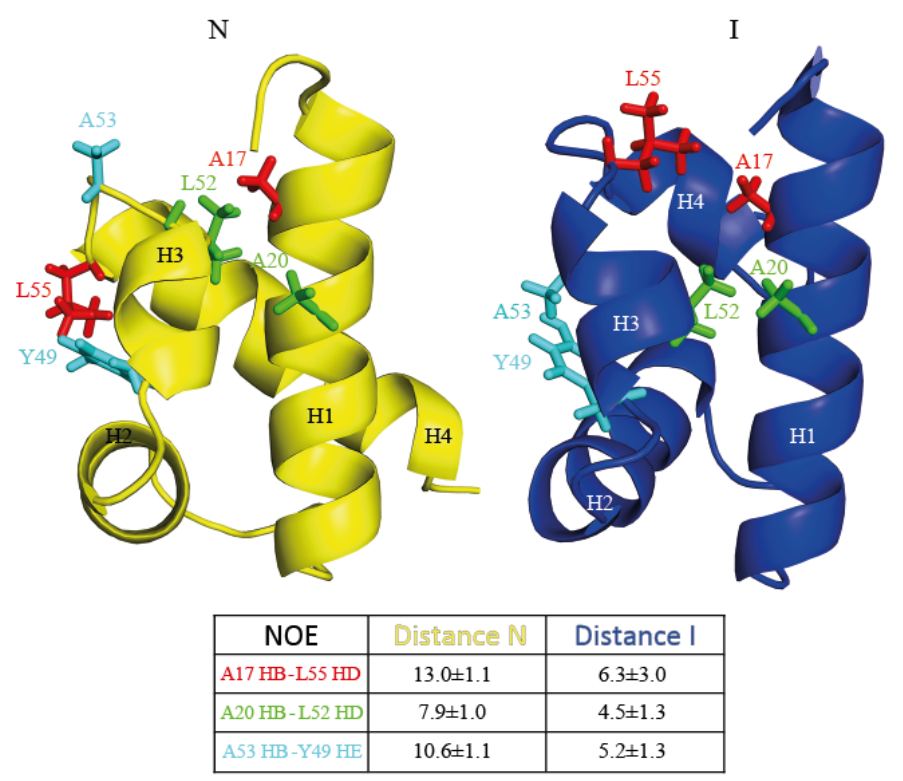

(b)

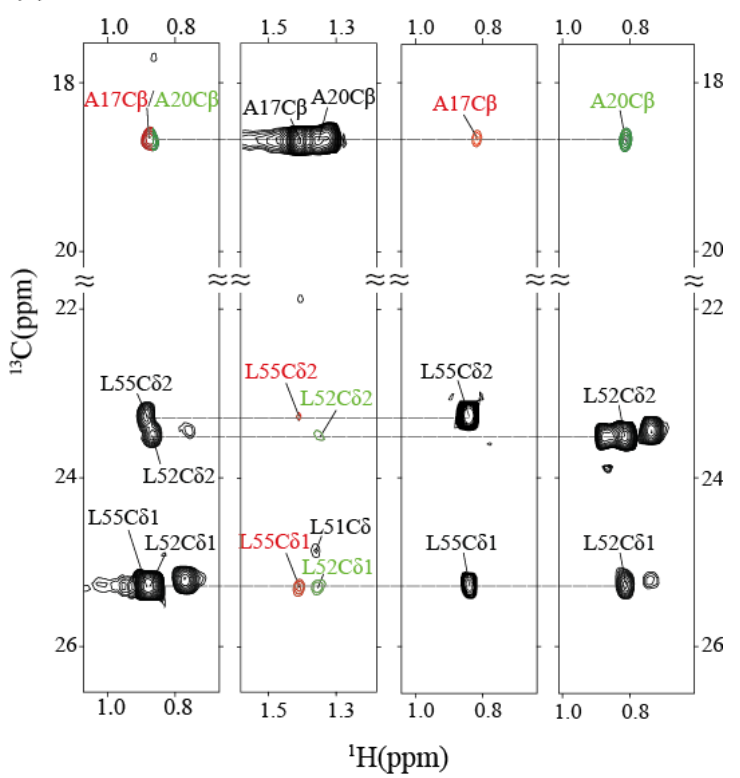

(c)

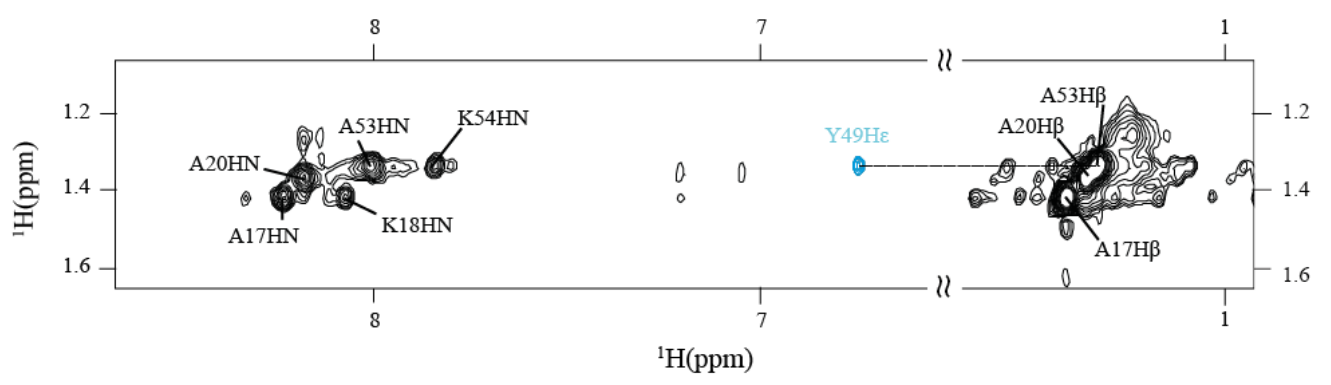

Figure 10. Non-native interactions predicted from the NMR relaxation dispersion/CS-Rosetta structure of I[1] are present in I'. (a) Structures of N (pdb accession code 1UZC) and I (pdb accession code 2KZG) states of FF $_{1-71}$. Coloured in red, green and cyan are three pairs of residues that form non-native interactions in I, with the average and standard deviation of the distance between each residue pair in the native and intermediate states indicated in tabular form. Also indicated in the table is the Leu55H $\delta-T y r 49 H \varepsilon$ distance in I and N. (b) $\mathrm{F}_{1}-\mathrm{F}_{3}$ strip-plots from a constant-time methyl-methyl NOESY spectrum[80] of $\mathrm{FF}_{11-60}\left(25^{\circ} \mathrm{C}, 150 \mathrm{~ms}\right.$ mixing time $)$. NOE cross-peaks between L55 H $\delta 1 / 2$ - A17 H $\beta$ are shown in red and between L52 H $\delta 1 / 2$ - A20 H $\beta$ in green. (c) Strip plot from a NOESY spectrum of $\mathrm{FF}_{11-60}$ correlating Ala ${ }^{1} \mathrm{H} \beta /{ }^{13} \mathrm{C} \beta$ chemical shifts with ${ }^{1} \mathrm{H}$ shifts of proximal protons $\left(25^{\circ} \mathrm{C}, 250\right.$ ms mixing time, see Figure 4). An NOE cross-peak between A53 H $\beta$ and Y49 He is shown in cyan.

An NOE connecting Y49/L55 was reported for the native structure that was not observed in data sets recorded for I', again consistent with the expected distances in each state (Figure 10a). The 
observation of non-native NOEs in spectra of $\mathrm{I}^{\prime}$, predicted from the structural model of I, provides very strong support of the CS-Rosetta based structure of the 'invisible' folding intermediate of the FF domain.

\subsection{The solution structure of I' validates the CS-Rosetta structure of I}

Having established that I and I' have similar secondary structures and that they both share the same non-native contacts, we next solved the structure of I' using the standard NOE-directed approach (see Figure 6 for structural statistics). A superposition of the 10 lowest energy structures with no violations is shown in Figure 11a, with a single representative structure highlighting the non-native interactions discussed in the previous section and color coded as in Figure 10 illustrated in Figure 11b. These non-native distances for I' are tabulated in the figure. Notably, they are all within one standard deviation of the corresponding mean distances calculated for the CS-Rosetta structure of the folding intermediate (differences less than $1.5 \AA$ ), but are at least 2 standard deviations away from the corresponding mean distances computed for the native state structure (differences ranging between 2.5 - $7 \AA$ ). Figure 11c shows the superposition of the ensemble comprising the 10 lowest energy I state structures in blue (pdb code $2 \mathrm{KZG}$; pairwise RMSD including all backbone heavy atoms $=1.1 \pm 0.5 \AA$ ) with the corresponding ten 'best' structures calculated for I' in green (pair wise RMSD $=0.8 \pm 0.2 \AA$ ). The backbone RMSD of the two structural ensembles excluding loops and termini (residues 14-28, $36-45$ and 47-55) is $1.7 \pm 0.3 \AA$, climbing to $2.1 \pm 0.3 \AA$ when these regions are included in the calculation. Typically, structures of proteins smaller than approximately 120 residues calculated using CS-Rosetta have a backbone RMSD of 1-2 A relative to the experimentally determined Xray or NMR structure [48, 49]. The RMSD noted here, on the high end, likely reflects the fact that while I' appears to be an excellent mimic of the wild-type folding intermediate, there are differences in the molecules that are compared (residues 11-60 vs 1-71) that could well lead to subtle changes in structure. For example, the absence of $\mathrm{H} 4$ in the deletion mutant, $\mathrm{FF}_{11-60}$, may lead to structural perturbations relative to the intermediate since in the structure of I there are contacts formed between H4 (that is only partially formed) and H2[1]. In turn this could account for the slight differences in the orientation of helices between the two ensembles. Other differences may simply reflect the quality of the I' structure that is compromised by a reduced number of NOE constraints due to the dynamical nature of $\mathrm{FF}_{11-60}$ which results in broadening of 
residues Leu24, Lys28 and Arg29 in particular, and by the relatively small number of restraints used to calculate I via the CS-Rosetta approach. Nevertheless it is quite clear that the structures of I and I' are very similar and that the intermediate conformation reported previously which uses only CPMG-based restraints is accurate.

(a)

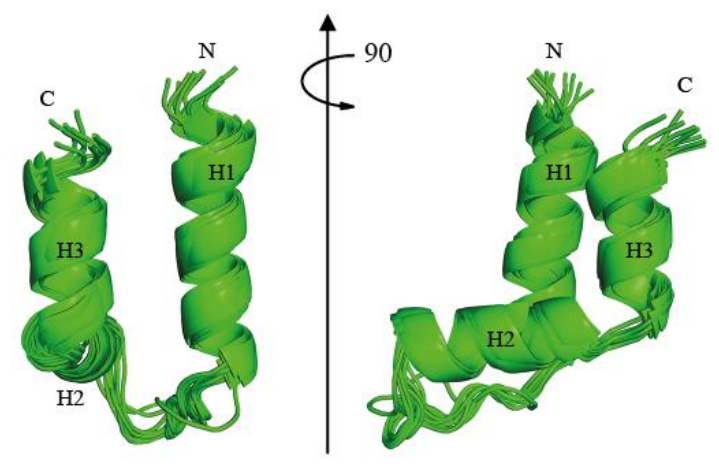

(c)

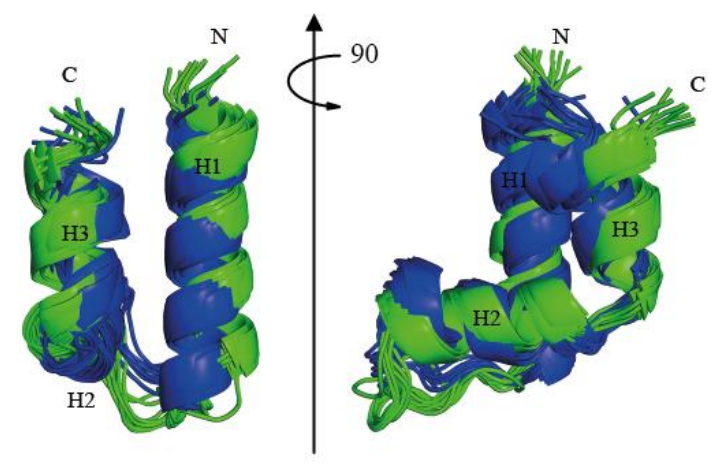

(b)

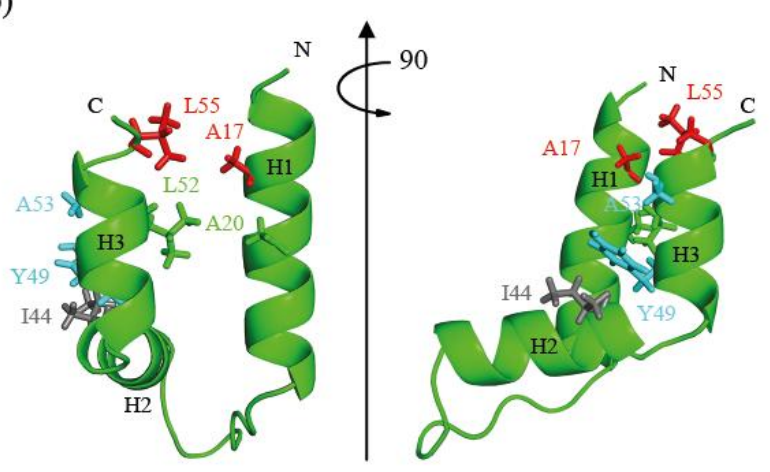

\begin{tabular}{|c|c|}
\hline NOE & Distance $(\AA)$ \\
\hline A17 HB -L55 Hô & $6.0 \pm 1.0$ \\
\hline $\mathrm{A} 20 \mathrm{H} \beta$ - L $52 \mathrm{H} \delta$ & $5.4 \pm 1.2$ \\
\hline A $53 \mathrm{HB}-\mathrm{Y} 49 \mathrm{H \varepsilon}$ & $5.6 \pm 1.1$ \\
\hline $\mathrm{L} 55 \mathrm{Ho}-\mathrm{Y} 49 \mathrm{H \varepsilon}$ & $11.4 \pm 1.5$ \\
\hline
\end{tabular}

Figure 11. Solution structure of the FF domain folding intermediate mimic, I'. (a) Superposition of the 10 lowest energy structures with no violations out of 100 calculated. The backbone heavy atom RMSD for the ensemble is $0.8 \pm 0.2 \AA$. (b) Lowest energy structure of I'; highlighted in red, green and cyan are three pairs of residues forming non-native interactions in the I state, with the average and standard deviation of the distance between each pair in I' indicated in the table. (c) Superposition of the ensemble of 10 representative lowest energy structures of I (blue, pdb code 2 KZG, rmsd of $1.1 \pm 0.5 \AA$ ) with the 10 lowest energy structures of I' (green). The backbone RMSD between pairs of structures from each of the ensembles is $2.1 \pm 0.3 \AA$. Only shown for I are those residues that are also present in I'. 


\section{Chapter 4}

\section{Conclusion}

\subsection{Validation of the relaxation dispersion method of solving excited state structures}

The development of CPMG relaxation dispersion NMR spectroscopy, in concert with new and powerful data-base computational approaches for structure determination based on chemical shift restraints, opens up the possibility of obtaining atomic resolution structures of excited protein states[1,31] that play important roles in protein folding[21], enzymology[72-74] and molecular recognition[75]. As with any new methodology the need for cross-validation is critical. Yet this is particularly challenging in studies of 'invisible' states because the options for probing detailed structure in such systems are limited. One approach is to use the structure of the excited state as a starting point for the rational design of a stabilized form of the molecule through carefully crafted mutations that would in turn destabilize what was formally the ground state. In this manner the relative populations of the ground and excited states become inverted, the excited state becomes 'visible' and traditional structural biology methods can then be used for cross validation. This is a powerful approach because mutagenesis creates a stable version of the state of interest that is likely to be well behaved for biophysical characterization. An alternative approach is one where structures of the native and excited states are compared so as to come up with potential mutations that would have little effect on the structure of the higher energy conformer, while preventing the ground state from forming. In this case the 'new' ground state (that is, the former excited state) can again be studied by standard structural biology techniques, as we have demonstrated for I' in this thesis. Such studies can be hampered, however, by the fact that the stability of this state remains essentially unchanged from that of the excited conformation, as illustrated in Figure 7.

This thesis details the use of mutagenesis to destabilize the FF native state and increase the folding intermediate-like state $\left(\mathrm{I}^{\prime}\right)$ to a level that is amenable to structure calculation by standard multi-dimensional solution state NMR methods. Notably, the structures of I' and I are similar, with the non-native contacts predicted by the CS-Rosetta model (I) observed in the I' conformation. This provides strong conformation of the previously proposed I state structure. 
Further, the work demonstrates the utility in using a combined NMR relaxation dispersion/CSRosetta approach for studies of 'invisible' excited protein states, providing structural data at a level of detail not possible using other biophysical techniques.

\subsection{Mechanism of FF folding}

The FF domain was postulated to fold by a nucleation-condensation mechanism based on $\varphi$ value analysis[54], as the region comprising the $\mathrm{C}$-terminus of $\mathrm{H} 1$, the $\mathrm{H} 1-\mathrm{H} 2$ loop and the $\mathrm{N}$ terminus of $\mathrm{H} 2$ forms native secondary and tertiary interactions in the major transition state while the remainder of the protein does not. The structure of the FF folding intermediate is indeed only completely native in the predicted region, but it also has fully native helices $\mathrm{H} 1$ and $\mathrm{H} 2$, which suggests a framework model. As previously mentioned, other $\alpha$-helical proteins have been determined (mainly by $\varphi$-value analysis) to fold by either a framework or nucleationcondensation mechanism $[54,56,58-60]$. It is thought that these two mechanisms are manifestations of a single mode of folding where decreasing stability of the secondary structure prompts a transition from framework to nucleation-condensation as tertiary interactions play a larger role in stabilizing the intermediate and transition states[61]. From our high-resolution structure of the FF folding intermediate, it seems that its mechanism of folding is indeed intermediate between the two models. However neither of these traditional models of protein folding takes into account the formation of non-native interactions in the intermediate state.

Instead of resorting to models, one can use the structure of the FF intermediate state to perform a detailed analysis of FF folding. The AGADIR program[93-95] calculates the residue-wise helical propensity of a polypeptide chain based on an empirical scale of propensity by amino acid type[96]. AGADIR predicts low overall intrinsic helical propensity for the FF domain ( 2\%). The highest-propensity helix is $\mathrm{H} 1$ at $\sim 4.5 \%$, followed by $\mathrm{H} 3$ of the intermediate $\left(\mathrm{H} 3^{\mathrm{I}}\right)$ at $\sim 4 \%$, $\mathrm{H} 2$ and $\mathrm{H} 3$ of the native state $\left(\mathrm{H} 3^{\mathrm{N}}\right.$ ) at $\sim 2.5 \%$ (although $\mathrm{H} 3{ }^{\mathrm{N}}$ is even less favorable as it adopts the less stable $3_{10}$ conformation) and finally $\mathrm{H}^{\mathrm{N}}$ at $\sim 1.5 \%$ (Figure 13). Based on the intrinsic helical propensities, it seems that the initial collapse of the unfolded polypeptide occurs when the three regions with highest helical propensity $\left(\mathrm{H} 1, \mathrm{H} 2\right.$ and $\left.\mathrm{H} 3^{\mathrm{I}}\right)$ form helices, which must be stabilized by (non-native) tertiary interactions due to their low intrinsic helical propensity. However the folding intermediate thus created has an unfolded helix $\mathrm{H} 4$ which leaves many hydrophobic residues exposed to solvent. As a consequence of this, helical propensity is 
overridden by the hydrophobic effect and $\mathrm{H} 3^{\mathrm{I}}$ unfolds to $\mathrm{H} 3^{\mathrm{N}}$ to allow the formation of a larger, more stable hydrophobic core involving H4. It seems therefore that the disconnect between optimal hydrophobic burial and intrinsic helical propensity leads to the formation of non-native secondary and tertiary interactions which in turn leads to the accumulation of a folding intermediate.

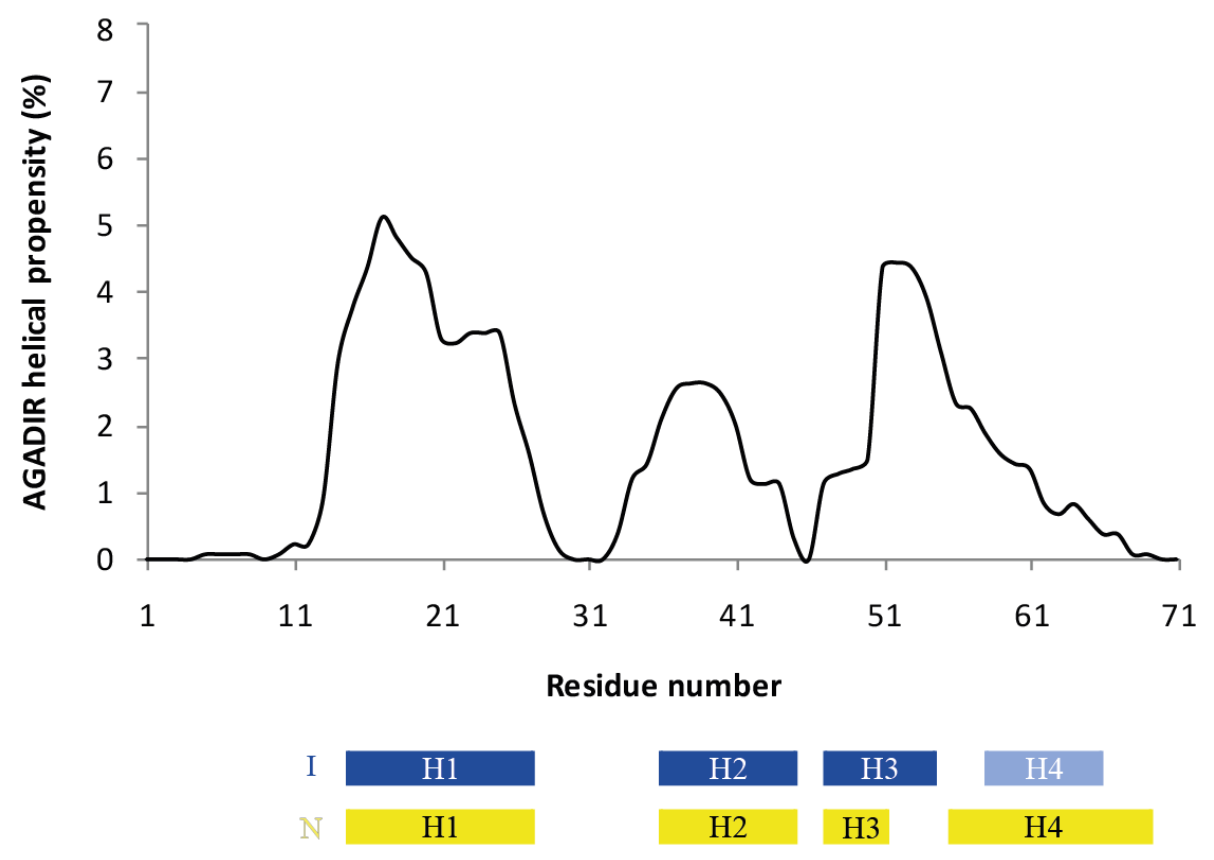

Figure 12. Per-residue helical propensity for the FF domain as predicted by AGADIR. The standard conditions for the study of $\mathrm{FF}_{1-71}(\mathrm{pH} 5.7,298.15 \mathrm{~K}, 0.15 \mathrm{M}$ ionic strength) were used for the prediction[1, 9, 54]. Helices are shown as rectangles. They span residues 14-28 (H1), 36-44 $(\mathrm{H} 2)$ and 47-54 $\left(\mathrm{H} 3^{\mathrm{I}}\right)$, with partial helicity in the 57-65 region for I. For N they span 14-28 (H1), 36-44 (H2), 47-51 $\left(\mathrm{H}^{\mathrm{N}}\right)$ and 55-69 $(\mathrm{H} 4)$.

\subsection{Non-native interactions in the FF folding intermediate}

The FF intermediate forms multiple non-native interactions involving $\mathrm{H} 3{ }^{\mathrm{I}}[1]$. Many structures of folding intermediates solved to date show non-native tertiary interactions, and the engrailed homeodomain also shows non-native helical boundaries[27, 55-57]. Previous studies of the FF domain have shown that the conversion from the intermediate to the native state is rate limiting[9]. Likely, it is the breaking of the non-native interactions that stabilize the intermediate which accounts for the large energy barrier of rearrangement. Non-native interactions have often been shown to play an important role in controlling the kinetics and thermodynamics of the folding pathway. They either increase the folding rate by stabilizing the transition state, or decrease the rate of folding by trapping the protein in a stable, non-native conformation. The FF 
domain is affected by the second phenomenon. The involvement of non-native interactions in slowing folding is further supported by the folding behavior of an FF domain L24A point mutant[32]. The L24A folding intermediate forms even more extensive non-native interactions than the wild type intermediate despite having an identical native fold, and consequently has a folding rate which is 7 times slower.

A popular theory is that proteins have evolved for function, not folding speed, and that nonnative interactions may arise by chance from a primary sequence designed for functionality (reviewed in [14]). For example, in the Pin1 WW[97] and Fyn SH3[98] domains, the replacement of sequence elements that are essential for function with sequence elements that have a high (non-native) secondary structure propensity which stabilises the transition state increases the rate of folding dramatically. In the FF domain, residues Ala 20 and Leu 52, which form one of the rate-limiting non-native interactions in the intermediate, are also critical to the stability of the native state. $\Phi$-value analysis showed that mutating either of these residues leads to a reduction in stability of the native state by around $3 \mathrm{kcal} / \mathrm{mol}[54]$. Non-native interactions may also be selected for to avoid aggregation[14].

Looking at the question from the opposite side of the folding pathway, it has also been shown that in the unfolded state a protein may have a predisposition to form some interactions involved in the native structure, but it may also have a predisposition to form other non-native energyminimizing interactions[99-101]. This has been observed for example in the drkN SH3 domain whose unfolded state can easily be studied as it exists in approximately $1: 1$ equilibrium with the folded state under non-denaturing conditions, and which forms a compact ensemble with native and non-native structure[101]. From this unfolded ensemble, it seems likely that transient intermediates displaying non-native interactions would arise. The extent and role of non-native interactions remains unclear, but what is clear is that they are very common and have a large impact on the kinetics of folding. 


\section{Chapter 5 \\ Future directions}

Ashok Sekhar compiled the list of proteins and their mutants that display the appropriate range of parameters for relaxation dispersion studies (Figure 13).

To understand how a protein transitions from an elongated polypeptide to its folded form (the protein folding problem), one would need a complete picture of all of states adopted by the protein during folding. It is very difficult experimentally to obtain detailed structures of all but the lowest-energy native state of the protein. The relaxation dispersion method provides a means of obtaining atomic-resolution information for folding intermediate states and as a result the folding pathway of the FF domain can now be understood to a large extent. All of the rates of interconversion and relative energies between states are known[9, 53], the structures of I[1] and $\mathrm{N}[62]$ have been solved, and the structure of the rate-limiting transition state between I and $\mathrm{N}$ has been thoroughly probed by $\varphi$-value analysis[54]. However the structures of the transition state between $\mathrm{U}$ and $\mathrm{I}$ and of the unfolded ensemble $\mathrm{U}$ are missing. The structure of the transition state between $U$ and I could be probed by $\Phi$-value analysis of the intermediate-state mimic, $I^{\prime}$. The unfolded ensemble could be characterized by a hybrid experimental/computational approach using structural restraints obtained by relaxation dispersion from I' which exchanges with an appropriate population of $\mathrm{U}(\sim 8 \%)$, providing of course that the timescale of exchange between $\mathrm{I}^{\prime}$ and $U$ is within the correct range $(\sim 50 \mu \mathrm{s}-10 \mathrm{~ms})$.

There are currently only three methods available to study folding at the atomic resolution: NMR to study equilibrium intermediates and denatured states in solution, $\varphi$-values analysis to give details of the structures of transition states, and atomic-level molecular dynamics (MD) simulation for a complete, all-atom picture of the folding pathway (reviewed in [102]). Currently, a combination of experiment and simulation can be used to describe folding at atomic resolution, a prime example being folding of the Engrailed homeodomain[7]. It is essential to benchmark simulations by experiment because the potential functions they employ are empirical and contain approximations[102]. The technique of relaxation dispersion provides a means of benchmarking MD simulations as it provides data on intermediate states which can be used to validate intermediates predicted by simulation. For example, relaxation dispersion results on the 
folding intermediate state of an SH3 domain are in close agreement with the structure of the first transition state of a very similar SH3 domain as predicted by simulation[52]. Furthermore, once the simulation has been corroborated by experiment, it can be used to fill in any missing folding events[102]. In other words, there is synergy between experiment and simulation: all-atom MD simulations provide a molecular framework for the interpretation of experiment which in turn can serve to validate the simulations. MD Simulations would be quite useful to further our understanding of FF domain folding by filling in the events which lead to the formation of the intermediate state.

Relaxation dispersion is applicable not only to productive, on-pathway folding intermediates, but also to off-pathway intermediates which may have a role in diseases of protein aggregation such as Alzheimer's disease, Parkinson's disease, Huntington's disease, amyotrophic lateral sclerosis and prion diseases (reviewed in [11]). Hydrophobic side-chains that, in the native state, are buried in the protein core are solvent exposed in the intermediate, making them accessible for intermolecular interactions that lead to aggregation. Therefore, solving the structure of excited states involved in folding by relaxation dispersion NMR could not only help to solve the protein folding problem but could also provide clues to the molecular basis for misfolding and aggregation.

Finally, the technique can be applied to proteins whose catalytic or ligand-binding conformation is only transiently populated. These biologically important excited states are difficult if not impossible to study by any other means. Despite the population and exchange timescale restrictions, it has already been shown that the excited states of many proteins with varied folds such as the all- $\alpha$-helical apomyoglobin[103], the all- $\beta$-sheet Fyn SH3 domain[21] and the integral membrane protein phospholamban[104] can be studied in detail by relaxation dispersion. Furthermore, other proteins and their mutants have been shown by $\varphi$-value analysis to be within the appropriate range of excited state population (above 0.5\%) and exchange rate (between 100 and $2000 \mathrm{~s}^{-1}$ ) for dispersion measurements. Some examples are included in Figure 13, and future experiments should uncover still more. The structure of these excited states can now confidently be solved using the relaxation dispersion/CS-Rosetta approach. 


\begin{tabular}{|c|c|c|c|}
\hline Protein & Mutants & $\begin{array}{c}\text { Excited state } \\
\text { population }(\%)\end{array}$ & $\begin{array}{c}\text { Exchange rate } \\
\left(\mathrm{s}^{-1}\right)\end{array}$ \\
\hline \multirow[t]{6}{*}{ Human procarboxypeptidase A2[105] } & $\mathrm{V} 12 \mathrm{~A}$ & 1.21 & 365.8 \\
\hline & F39L & 2.08 & 529.0 \\
\hline & A50G & 1.10 & 561.7 \\
\hline & F65A & 0.84 & 194.1 \\
\hline & I71V & 0.81 & 449.5 \\
\hline & $\mathrm{I} 75 \mathrm{~A}$ & 1.37 & 334.9 \\
\hline \multirow{12}{*}{$\begin{array}{c}\text { Cold shock protein from Bacillus } \\
\text { caldolyticus }[106]\end{array}$} & L3E & 0.95 & 481.6 \\
\hline & $\mathrm{K} 5 \mathrm{~A}$ & 1.66 & 166.8 \\
\hline & K7A & 1.00 & 196.0 \\
\hline & N10A & 0.87 & 261.3 \\
\hline & F15A & 3.37 & 208.0 \\
\hline & F17A & 1.14 & 923.5 \\
\hline & I18V & 4.05 & 774.4 \\
\hline & V26T & 1.78 & 180.2 \\
\hline & $\mathrm{I} 33 \mathrm{~A}$ & 7.42 & 1112.5 \\
\hline & L41A & 8.02 & 279.4 \\
\hline & I51A & 4.48 & 732.8 \\
\hline & A60G & 7.28 & 685.9 \\
\hline Sso7d from Sulfolobus solfataricus [107] & L55A & 3.08 & 257.6 \\
\hline Human ubiquitin[108] & L43A & 1.11 & 455.4 \\
\hline $\begin{array}{c}\text { N-terminal of ribosomal protein L9 from } \\
\text { Bacillus stearothermophilus[109] }\end{array}$ & WT & 3.03 & 660.0 \\
\hline
\end{tabular}

Figure 13. Examples of proteins and their mutants that display the appropriate range of parameters for relaxation dispersion studies. $\Phi$-value analysis indicates that for each one the excited state population is above $0.5 \%$, and the exchange rate is between 100 and $20000 \mathrm{~s}^{-1}$, as required for relaxation dispersion. 


\section{References}

1. Korzhnev, D.M., et al., A transient and low-populated protein-folding intermediate at atomic resolution. Science, 2010. 329(5997): p. 1312-1316.

2. Sela, M., White, F.H. Jr and Anfinsen, C.B., Reductive cleavage of disulfide bridges in ribonuclease Science, 1957. 125(3250): p. 691-2.

3. Anfinsen, C.B., Principles that govern the folding of protein chains. Science, 1973. 181(96): p. 223-30.

4. Levinthal, C. How to Fold Graciously. in Mossbauer Spectroscopy in Biological Systems. 1969. Allerton House, Monticello, Illinois: University of Illinois Press.

5. Levinthal, C., Are there pathways for protein folding? J Chem Phys, 1968. 65: p. 44-45.

6. Fersht, A.R., A kinetically significant intermediate in the folding of barnase. Proc Natl Acad Sci U S A, 2000. 97(26): p. 14121-6.

7. Mayor, U., et al., The complete folding pathway of a protein from nanoseconds to microseconds. Nature, 2003. 421(6925): p. 863-7.

8. Capaldi, A.P., et al., Ultrarapid mixing experiments reveal that Im 7 folds via an onpathway intermediate. Nat Struct Biol, 2001. 8(1): p. 68-72.

9. Jemth, P., et al., Demonstration of a low-energy on-pathway intermediate in a fastfolding protein by kinetics, protein engineering, and simulation. Proc Natl Acad Sci U S A, 2004. 101(17): p. 6450-5.

10. Religa, T.L., et al., Solution structure of a protein denatured state and folding intermediate. Nature, 2005. 437(7061): p. 1053-6.

11. Chiti, F. and C.M. Dobson, Amyloid formation by globular proteins under native conditions. Nat Chem Biol, 2009. 5(1): p. 15-22.

12. Kim, P.S. and R.L. Baldwin, Specific intermediates in the folding reactions of small proteins and the mechanism of protein folding. Annu Rev Biochem, 1982. 51: p. 459-89.

13. Auer, S., et al., Importance of metastable states in the free energy landscapes of polypeptide chains. Phys Rev Lett, 2007. 99(17): p. 178104.

14. Brockwell, D.J. and S.E. Radford, Intermediates: ubiquitous species on folding energy landscapes? Curr Opin Struct Biol, 2007. 17(1): p. 30-7.

15. Daggett, V. and A. Fersht, The present view of the mechanism of protein folding. Nat Rev Mol Cell Biol, 2003. 4(6): p. 497-502. 
16. Dill, K.A. and D. Shortle, Denatured states of proteins. Annu Rev Biochem, 1991. 60: p. 795-825.

17. Dinner, A.R., et al., Understanding protein folding via free-energy surfaces from theory and experiment. Trends Biochem Sci, 2000. 25(7): p. 331-9.

18. Dill, K.A., Theory for the folding and stability of globular proteins. Biochemistry, 1985. 24(6): p. 1501-9.

19. Wetlaufer, D.B., Nucleation, rapid folding, and globular intrachain regions in proteins. Proc Natl Acad Sci U S A, 1973. 70(3): p. 697-701.

20. Jackson, S.E. and A.R. Fersht, Folding of chymotrypsin inhibitor 2. 1. Evidence for a two-state transition. Biochemistry, 1991. 30(43): p. 10428-35.

21. Korzhnev, D.M., et al., Low-populated folding intermediates of Fyn SH3 characterized by relaxation dispersion NMR. Nature, 2004. 430(6999): p. 586-90.

22. Bai, Y., Kinetic evidence for an on-pathway intermediate in the folding of cytochrome $c$. Proc Natl Acad Sci U S A, 1999. 96(2): p. 477-80.

23. Chu, R., et al., Relationship between the native-state hydrogen exchange and folding pathways of a four-helix bundle protein. Biochemistry, 2002. 41(25): p. 7998-8003.

24. Takei, J., et al., Populating partially unfolded forms by hydrogen exchange-directed protein engineering. Biochemistry, 2002. 41(41): p. 12308-12.

25. Hollien, J. and S. Marqusee, Comparison of the folding processes of T. thermophilus and E. coli ribonucleases $H$. J Mol Biol, 2002. 316(2): p. 327-40.

26. Feng, H., et al., Specific non-native hydrophobic interactions in a hidden folding intermediate: implications for protein folding. Biochemistry, 2003. 42(43): p. 12461-5.

27. Feng, H., Z. Zhou, and Y. Bai, A protein folding pathway with multiple folding intermediates at atomic resolution. Proc Natl Acad Sci U S A, 2005. 102(14): p. 5026-31.

28. Kato, H., H. Feng, and Y. Bai, The folding pathway of T4 lysozyme: the high-resolution structure and folding of a hidden intermediate. J Mol Biol, 2007. 365(3): p. 870-80.

29. Zhou, Z., et al., The high-resolution NMR structure of the early folding intermediate of the Thermus thermophilus ribonuclease H. J Mol Biol, 2008. 384(2): p. 531-9.

30. Hansen, D.F., P. Vallurupalli, and L.E. Kay, Using relaxation dispersion NMR spectroscopy to determine structures of excited, invisible protein states. J Biomol NMR, 2008. 41(3): p. 113-20.

31. Vallurupalli, P., D.F. Hansen, and L.E. Kay, Structures of invisible, excited protein states by relaxation dispersion NMR spectroscopy. Proc Natl Acad Sci U S A, 2008. 105(33): p. 11766-71. 
32. Korzhnev, D.M., et al., Nonnative Interactions in the FF Domain Folding Pathway from an Atomic Resolution Structure of a Sparsely Populated Intermediate: An NMR Relaxation Dispersion Study. J Am Chem Soc, 2011.

33. Carr, H.Y. and E.M. Purcell, Effects of diffusion on free precession in nuclear magnetic resonance experiments. Phys. Rev., 1954. 54: p. 630-638.

34. Meiboom, S. and D. Gill, Modified spin-echo method for measuring nuclear magnetic relaxation times. Rev. Sci. Instrum., 1958. 29: p. 688-691.

35. Palmer, A.G., 3rd, C.D. Kroenke, and J.P. Loria, Nuclear magnetic resonance methods for quantifying microsecond-to-millisecond motions in biological macromolecules. Methods Enzymol, 2001. 339: p. 204-38.

36. Korzhnev, D.M. and L.E. Kay, Probing invisible, low-populated States of protein molecules by relaxation dispersion NMR spectroscopy: an application to protein folding. Acc Chem Res, 2008. 41(3): p. 442-51.

37. Skrynnikov, N.R., F.W. Dahlquist, and L.E. Kay, Reconstructing NMR spectra of "invisible" excited protein states using HSQC and HMQC experiments. J. Am. Chem. Soc., 2002. 124(41): p. 12352-60.

38. Auer, R., et al., Measuring the signs of 1 H(alpha) chemical shift differences between ground and excited protein states by off-resonance spin-lock R(1rho) NMR spectroscopy. J Am Chem Soc, 2009. 131(31): p. 10832-3.

39. Bouvignies, G., et al., A simple method for measuring signs of $(1) H(N)$ chemical shift differences between ground and excited protein states. J Biomol NMR, 2010. 47(2): p. 135-41.

40. Loria, J.P., M. Rance, and A.G. Palmer, A relaxation compensated CPMG sequence for characterizing chemical exchange. J. Am. Chem. Soc., 1999. 121: p. 2331-2332.

41. Tollinger, M., et al., Slow dynamics in folded and unfolded states of an SH3 domain. J. Am. Chem. Soc., 2001. 123: p. 11341-11352.

42. Ishima, R. and D. Torchia, Extending the range of amide proton relaxation dispersion experiments in proteins using a constant-time relaxation-compensated CPMG approach. J. Biomol. NMR, 2003. 25: p. 243-248.

43. Hansen, D.F., et al., Probing Chemical Shifts of Invisible States of Proteins with Relaxation Dispersion NMR Spectroscopy: How Well Can We Do? J. Am. Chem. Soc., 2008. 130: p. 2667-2675.

44. Ishima, R., et al., Carbonyl carbon transverse relaxation dispersion measurements and ms-micros timescale motion in a protein hydrogen bond network. J. Biomol. NMR, 2004. 29(2): p. 187-98. 
45. Lundstrom, P., D.F. Hansen, and L.E. Kay, Measurement of carbonyl chemical shifts of excited protein states by relaxation dispersion NMR spectroscopy: comparison between uniformly and selectively (13)C labeled samples. J Biomol NMR, 2008. 42(1): p. 35-47.

46. Lundstrom, P., et al., Accurate measurement of alpha proton chemical shifts of excited protein states by relaxation dispersion NMR spectroscopy. J Am Chem Soc, 2009.

131(5): p. 1915-26.

47. Vallurupalli, P., et al., Measurement of bond vector orientations in invisible excited states of proteins. Proc Natl Acad Sci U S A, 2007. 104(47): p. 18473-7.

48. Shen, Y., et al., Consistent blind protein structure generation from NMR chemical shift data. Proc. Natl. Acad. Sci. U.S.A., 2008. 105(12): p. 4685-90.

49. Cavalli, A., et al., Protein structure determination from NMR chemical shifts. Proc. Natl. Acad. Sci. U.S.A., 2007. 104(23): p. 9615-20.

50. Wishart, D.S., et al., CS23D: a web server for rapid protein structure generation using NMR chemical shifts and sequence data. Nucleic Acids Res, 2008. 36(Web Server issue): p. W496-502.

51. Raman, S., et al., NMR structure determination for larger proteins using backbone-only data. Science. 327(5968): p. 1014-8.

52. Mittermaier, A., D.M. Korzhnev, and L.E. Kay, Side-chain interactions in the folding pathway of a Fyn SH3 domain mutant studied by relaxation dispersion NMR spectroscopy. Biochemistry, 2005. 44(47): p. 15430-6.

53. Korzhnev, D.M., et al., The folding pathway of an FF domain: characterization of an onpathway intermediate state under folding conditions by (15)N, (13)C(alpha) and (13)Cmethyl relaxation dispersion and (1)H/(2)H-exchange NMR spectroscopy. J Mol Biol, 2007. 372(2): p. 497-512.

54. Jemth, P., et al., The structure of the major transition state for folding of an FF domain from experiment and simulation. J Mol Biol, 2005. 350(2): p. 363-78.

55. Wensley, B.G., et al., Experimental evidence for a frustrated energy landscape in a three-helix-bundle protein family. Nature. 463(7281): p. 685-8.

56. Capaldi, A.P., C. Kleanthous, and S.E. Radford, Im7 folding mechanism: misfolding on a path to the native state. Nat Struct Biol, 2002. 9(3): p. 209-16.

57. McCully, M.E., et al., Refolding the engrailed homeodomain: structural basis for the accumulation of a folding intermediate. Biophys J. 99(5): p. 1628-36.

58. Scott, K.A., L.G. Randles, and J. Clarke, The folding of spectrin domains II: phi-value analysis of R16. J Mol Biol, 2004. 344(1): p. 207-21. 
59. Scott, K.A., et al., The folding pathway of spectrin R17 from experiment and simulation: using experimentally validated MD simulations to characterize States hinted at by experiment. J Mol Biol, 2006. 359(1): p. 159-73.

60. Religa, T.L., et al., The helix-turn-helix motif as an ultrafast independently folding domain: the pathway of folding of Engrailed homeodomain. Proc Natl Acad Sci U S A, 2007. 104(22): p. 9272-7.

61. Daggett, V. and A.R. Fersht, Is there a unifying mechanism for protein folding? Trends Biochem Sci, 2003. 28(1): p. 18-25.

62. Allen, M., et al., The structure of an FF domain from human HYPA/FBP11. J Mol Biol, 2002. 323(3): p. 411-6.

63. Phatnani, H.P. and A.L. Greenleaf, Phosphorylation and functions of the RNA polymerase II CTD. Genes Dev, 2006. 20(21): p. 2922-36.

64. Corden, J.L., Tails of RNA polymerase II. Trends Biochem Sci, 1990. 15(10): p. 383-7.

65. Payne, J.M., P.J. Laybourn, and M.E. Dahmus, The transition of RNA polymerase II from initiation to elongation is associated with phosphorylation of the carboxyl-terminal domain of subunit IIa. J Biol Chem, 1989. 264(33): p. 19621-9.

66. Cadena, D.L. and M.E. Dahmus, Messenger RNA synthesis in mammalian cells is catalyzed by the phosphorylated form of RNA polymerase II. J Biol Chem, 1987. 262(26): p. 12468-74.

67. Fabrega, C., et al., Structure of an mRNA capping enzyme bound to the phosphorylated carboxy-terminal domain of RNA polymerase II. Mol Cell, 2003. 11(6): p. 1549-61.

68. Licatalosi, D.D., et al., Functional interaction of yeast pre-mRNA 3' end processing factors with RNA polymerase II. Mol Cell, 2002. 9(5): p. 1101-11.

69. Kim, E., et al., Splicing factors associate with hyperphosphorylated RNA polymerase II in the absence of pre-mRNA. J Cell Biol, 1997. 136(1): p. 19-28.

70. Misteli, T. and D.L. Spector, RNA polymerase II targets pre-mRNA splicing factors to transcription sites in vivo. Mol Cell, 1999. 3(6): p. 697-705.

71. Cho, H., et al., A human RNA polymerase II complex containing factors that modify chromatin structure. Mol Cell Biol, 1998. 18(9): p. 5355-63.

72. Boehr, D.D., et al., The dynamic energy landscape of dihydrofolate reductase catalysis. Science, 2006. 313(5793): p. 1638-42.

73. Fraser, J.S., et al., Hidden alternative structures of proline isomerase essential for catalysis. Nature, 2009. 462(7273): p. 669-73. 
74. Henzler-Wildman, K.A., et al., A hierarchy of timescales in protein dynamics is linked to enzyme catalysis. Nature, 2007. 450(7171): p. 913-6.

75. Korzhnev, D.M., et al., Alternate binding modes for a ubiquitin-SH3 domain interaction studied by NMR spectroscopy. J Mol Biol, 2009. 386(2): p. 391-405.

76. Sattler, M., J. Schleucher, and C. Griesinger, Heteronuclear multidimensional NMR experiments for the structure determination of proteins in solution employing pulsed field gradients. Prog. Nucl. Magn. Reson. Spectrosc., 1999. 34: p. 93-158.

77. Bax, A., Multidimensional nuclear magnetic resonance methods for protein studies. Curr. Opin. Struct. Biol., 1994. 4: p. 738-744.

78. Pascal, S., et al., Simultaneous acquisition of ${ }^{15} N$ and ${ }^{13} C$ edited NOE spectra of proteins dissolved in $\mathrm{H}_{2} \mathrm{O}$. J. Magn. Reson. Series B, 1994. 101: p. 197-201.

79. Neri, D., et al., Stereospecific nuclear magnetic resonance assignments of the methyl groups of valine and leucine in the DNA-binding domain of the 434 repressor by biosynthetically directed fractional ${ }^{13} \mathrm{C}$ labeling. Biochemistry, 1989. 28: p. 7510-7516.

80. Zwahlen, C., et al., An NMR experiment for measuring methyl-methyl NOEs in ${ }^{13} \mathrm{C}$ labeled proteins with high resolution. J. Am. Chem. Soc., 1998. 120: p. 7617-7625.

81. Green, H.a.F., R., Band-selective radiofrequency pulses. J. Magn. Reson., 1991. 93: p. 93-141.

82. Kupce, E. and R. Freeman, Adiabatic pulses for wideband inversion and broadband decoupling. J. Magn. Reson., Ser. A., 1995. 115: p. 273-276.

83. Zhang, S. and D.G. Gorenstein, Bloch-Siegert shift compensated and cyclic irradiation sidebands eliminated, double-adiabatic homonuclear decoupling for $13 \mathrm{C}$ - and $15 \mathrm{~N}$ double-labeled proteins. J Magn Reson, 1998. 132(1): p. 81-7.

84. Marion, D., et al., Rapid recording of 2D NMR spectra without phase cycling. Application to the study of hydrogen exchange in proteins. J. Magn. Reson., 1989. 85: p. 393-399.

85. Shen, Y., et al., TALOS+: a hybrid method for predicting protein backbone torsion angles from NMR chemical shifts. J Biomol NMR, 2009. 44(4): p. 213-23.

86. Berjanskii, M.V. and D.S. Wishart, A simple method to predict protein flexibility using secondary chemical shifts. J Am Chem Soc, 2005. 127(43): p. 14970-1.

87. Schwieters, C.D., et al., The Xplor-NIH NMR molecular structure determination package. J Magn Reson, 2003. 160(1): p. 65-73.

88. Schwieters, C.D., Kuszewski, J.J. and Clore, G.M., Using Xplor-NIH for NMR molecular structure determination. Progr. NMR Spectroscopy 2006. 48: p. 47-62. 
89. Kuszewski, J., A.M. Gronenborn, and G.M. Clore, Improvements and extensions in the conformational database potential for the refinement of NMR and X-ray structures of proteins and nucleic acids. J. Magn. Reson., 1997. 125: p. 171-177.

90. Laskowski, R.A., MacArthur, M.W., Moss, D.S., Thornton, J.M. , PROCHECK - a program to check the stereochemical quality of protein structures. J. App. Cryst., 1993. 26: p. 283-291.

91. Wishart, D.S., B.D. Sykes, and F.M. Richards, The chemical shift index: a fast and simple method for the assignment of protein secondary structure through NMR spectroscopy. Biochemistry, 1992. 31(6): p. 1647-51.

92. Spera, S. and A. Bax, An empirical correlation between protein backbone conformation and $\mathrm{Ca}$ and $\mathrm{Cb}$ chemical shifts. J. Am. Chem. Soc., 1991. 113: p. 5490-5492.

93. Lacroix, E., A.R. Viguera, and L. Serrano, Elucidating the folding problem of alphahelices: local motifs, long-range electrostatics, ionic-strength dependence and prediction of NMR parameters. J Mol Biol, 1998. 284(1): p. 173-91.

94. Munoz, V. and L. Serrano, Elucidating the folding problem of helical peptides using empirical parameters. Nat Struct Biol, 1994. 1(6): p. 399-409.

95. Munoz, V. and L. Serrano, Development of the multiple sequence approximation within the AGADIR model of alpha-helix formation: comparison with Zimm-Bragg and LifsonRoig formalisms. Biopolymers, 1997. 41(5): p. 495-509.

96. Munoz, V. and L. Serrano, Intrinsic secondary structure propensities of the amino acids, using statistical phi-psi matrices: comparison with experimental scales. Proteins, 1994. 20(4): p. 301-11.

97. Jager, M., et al., Structure-function-folding relationship in a WW domain. Proc Natl Acad Sci U S A, 2006. 103(28): p. 10648-53.

98. Di Nardo, A.A., et al., Dramatic acceleration of protein folding by stabilization of a nonnative backbone conformation. Proc Natl Acad Sci U S A, 2004. 101(21): p. 7954-9.

99. Shan, B., D. Eliezer, and D.P. Raleigh, The unfolded state of the C-terminal domain of the ribosomal protein $L 9$ contains both native and non-native structure. Biochemistry, 2009. 48(22): p. 4707-19.

100. Shan, B., et al., The cold denatured state of the C-terminal domain of protein L9 is compact and contains both native and non-native structure. J Am Chem Soc. 132(13): p. 4669-77.

101. Marsh, J.A., et al., Improved structural characterizations of the drkN SH3 domain unfolded state suggest a compact ensemble with native-like and non-native structure. $\mathrm{J}$ Mol Biol, 2007. 367(5): p. 1494-510. 
102. Fersht, A.R. and V. Daggett, Protein folding and unfolding at atomic resolution. Cell, 2002. 108(4): p. 573-82.

103. Meinhold, D.W. and P.E. Wright, Measurement of protein unfolding/refolding kinetics and structural characterization of hidden intermediates by NMR relaxation dispersion. Proc Natl Acad Sci U S A. 108(22): p. 9078-83.

104. Traaseth, N.J. and G. Veglia, Probing excited states and activation energy for the integral membrane protein phospholamban by NMR CPMG relaxation dispersion experiments. Biochim Biophys Acta. 1798(2): p. 77-81.

105. Villegas, V., et al., Structure of the transition state in the folding process of human procarboxypeptidase A2 activation domain. J Mol Biol, 1998. 283(5): p. 1027-36.

106. Magg, C. and F.X. Schmid, Rapid collapse precedes the fast two-state folding of the cold shock protein. J Mol Biol, 2004. 335(5): p. 1309-23.

107. Guerois, R. and L. Serrano, The SH3-fold family: experimental evidence and prediction of variations in the folding pathways. J Mol Biol, 2000. 304(5): p. 967-82.

108. Went, H.M. and S.E. Jackson, Ubiquitin folds through a highly polarized transition state. Protein Eng Des Sel, 2005. 18(5): p. 229-37.

109. Horng, J.C., V. Moroz, and D.P. Raleigh, Rapid cooperative two-state folding of a miniature alpha-beta protein and design of a thermostable variant. J Mol Biol, 2003. 326(4): p. 1261-70. 